\title{
The levitation of a sphere by two parallel turbulent jets
}

Thomas Barois, Guillaume Ricard, Victor Champain, Lucas Gey, and Hamid Kellay ${ }^{1}$

Univ. Bordeaux, CNRS, LOMA, UMR 5798, F-33405 Talence, France

Two vertical turbulent round jets are used to levitate a spherical particle. First, a symmetric configuration is investigated with the two jets of equal flow rates. A structure reminiscent of a pitchfork bifurcation is reported and characterized between a double trap configuration for low flow rates and a single trap regime at larger flow rates. A second configuration is then studied with the flow rates that differ between the left and the right jets. In this case, a preferential transition towards the jet of higher flow rate is reported and quantified. A model is presented predicting the force field for the symmetric and the asymmetric configurations. The model is compared to the force field measurements realized with a particle on a flexible cantilever. Finally, a particle dynamics simulation is proposed to analyze in more details the transition for the levitation in the symmetric and the asymmetric configurations.

\section{CONTENTS}

I. Introduction 2

II. Experimental setup 2

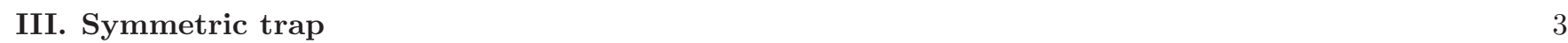

A. Transition from a double trap to a single trap 4

1. Trapping map 4

2. Particle altitude 5

\begin{tabular}{ll} 
3. Particle fluctuations & 6 \\
\hline
\end{tabular}

\begin{tabular}{ll}
\hline B. Rotation & 6
\end{tabular}

\begin{tabular}{|lr}
\hline IV. Force fields & 8 \\
\hline
\end{tabular}

\begin{tabular}{lr}
\hline A. Measurement & 9
\end{tabular}

\begin{tabular}{lr}
\hline B. Velocity and force summation rules & 10
\end{tabular}

$\begin{array}{ll}1 . \text { velocity field } & 10\end{array}$

2. Force field for a small sphere 11

\begin{tabular}{ll}
\hline 3. Force field for a large sphere & 11
\end{tabular}

V. Asymmetric trap 11

\begin{tabular}{ll}
\hline A. Trapping map & 12 \\
\hline
\end{tabular}

\begin{tabular}{ll}
\hline B. Topology of the trapping lines & 13
\end{tabular}

\begin{tabular}{|lr}
\hline VI. Particle dynamics simulation & 13
\end{tabular}

\begin{tabular}{ll}
\hline A. Equation of motion & 14
\end{tabular}

B. Particle dynamics simulation in the symmetric configuration 15

\begin{tabular}{ll}
\hline C. Particle dynamics in the asymmetric configuration: transition probability & 16
\end{tabular}

\begin{tabular}{lr}
\hline VII. Conclusion & 17
\end{tabular}

$\begin{array}{lr}\text { A. Force field: Empirical model 1 jet } & 17\end{array}$

\begin{tabular}{ll}
\hline 1. Measurement & 18
\end{tabular}

\begin{tabular}{lr}
\hline 2. Modal decomposition & 18
\end{tabular}

B. Calibration for the particle simulation 19

\begin{tabular}{ll}
\hline 1. Nature of the particle fluctuations & 20
\end{tabular}

\begin{tabular}{ll}
\hline 2. Damping & 20
\end{tabular}

\begin{tabular}{ll}
\hline 3. Noise & 20
\end{tabular} 


\section{INTRODUCTION}

The object of this work is to investigate the trapping and the dynamics of a spherical particle in levitation above two adjacent round jets. The purpose of this study is to evaluate if it is possible to control the position of a particle in levitation by a set of air jets without moving the jets nozzles. Two situations are addressed in this perspective. First, the location of a levitating particle is investigated in a configuration with the two jets of equal flow rate. Second, the motion of the particle is studied with a different flow rate for the two jets.

Among the realizations of levitation in physics 1 , the fluid levitation is probably the simplest to perform. The term

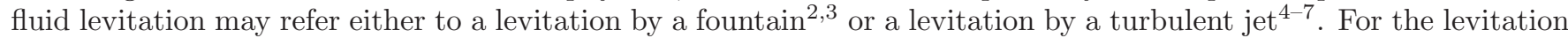
by a fountain, a water jet in air is typically used and the structure of the water jet is dominantly governed by gravity and surface tension, with little influence of the shear at the water/air interface. For turbulent jet levitation, only one fluid is involved and the jet structure is the result of an open shear flow.

In this study, only the fluid levitation with turbulent air jets is considered in a configuration for which the jets are vertical and with an ascending flow in order to levitate heavy object. The stability of a spherical particle in a single vertical turbulent jet is attributed to the Coandă effect $\stackrel{8}{\text { : }}$ with the sphere slightly off the jet axis, the deflection of the jet by the particle results in a net force oriented towards the jet axis $\stackrel{9}{ }$. The Coandă effect with monophasic jets is a high Reynolds number effect related to a momentum transfer involving the fluid inertia. The turbulent nature of a jet is not a priori required for the Coandă effect to exist but the levitation in monophasic vertical jets is practically observed in the turbulent regime. The levitation in non-turbulent jets is however possible with tilted jets $\underline{4}$ at moderate Reynolds numbers $R e \sim 10$. Inverted levitation is also possible in water experiments with jets pointing downward and buoyant particles 10,11 .

A turbulent jet in the high Reynolds number regime has a universal structure $\frac{12,13}{2}$. Except close to the nozzle $\frac{14,15}{2}$, the mean velocity profile is self-similar with a rescaling depending on the ratio of the radial distance with the distance to nozzle. The flow is maximal on the jet axis and it decreases proportionally to the inverse distance to the nozzle. The width of the jet is also proportional to the distance to the nozzle which gives a conical structure for a round jet.

For the levitation in a single jet, the equilibrium distance to the nozzle is a function of the flow rate. The flow rate has to be above a threshold related to the weight of the ball to allow levitation away from the nozzle. If the flow rate is increased above this threshold, the distance to the nozzle increases until the levitation state is lost because the fluctuations of the sphere are sufficient to release it. Note however that a non-contact state is possible well below the levitation threshold. This regime corresponds to suspension of the sphere in the lubrication limit with a very thin gap between the object and the nozzle. This strategy may be used to suppress thermal loses of heated objects $\underline{16}-\underline{18}$ or to get rid of the solid friction with heavy spheres $\frac{19}{}$. Also, vertical air flows may be used below the levitation threshold to investigate air-fluidized granular layers 20 .

In section II., the experimental setup used to capture a particle with two jets is presented. Then, a first configuration is investigated in section III. with the two jets having identical flow rates. Section IV. presents the measurement used to obtain a mapping of the force field. Section V. presents the experimental results obtained when the flow rate is different between the two jets. Finally a particle dynamics simulation approach is presented in section VI.

\section{EXPERIMENTAL SETUP}

The experiments are performed with air jets in an open room. The image in figure 1(a) presents a ping-pong ball levitating above two jets of equal flow rates. The diameter of the ball is $D=4 \mathrm{~cm}$, its mass is $m=2.7 \mathrm{~g}$. This configuration with two adjacent jets is a macroscopic equivalent of dual optical traps $21-25$.

The sketch in figure 1 (b) presents the structure of the connected tubes to obtain the two air jets. A first tube (inner diameter $10 \mathrm{~mm}$, length $3 \mathrm{~m}$ ) is connected to a pressure regulator. The input pressure is varied between 0 and 2 bars. After a connection to a tube of inner diameter $4 \mathrm{~mm}$, a T-junction is used to separate the flow in two branches $L$ and $R$ corresponding to left and right from the point of view of the camera. The tube lengths between the nozzle and the junction are $L_{L}$ for the left tube and $L_{R}$ for the right tube. The nozzles are metallic tubes with an inner diameter of $4 \mathrm{~mm}$. The tubes are mounted on rails and can be moved independently in the horizontal direction $x$. In all the configurations, the two nozzles have the same altitude $z=0$ and the origin $x=0, y=0$ and $z=0$ is set at the middle of the two nozzles.

The flow rates at the output of the nozzles are $Q_{L}$ for the left nozzle and $Q_{R}$ for the right nozzle. The values of $Q_{R}$ and $Q_{L}$ depend on the input pressure imposed at the regulator and the lengths $L_{L}$ and $L_{R}$. In the symmetric configuration $L_{L}$ equals $L_{R}$ and the flow rate is expected to be the same in both branches. In practice, a true symmetric configuration is difficult to obtain and the flow rates from the two nozzles are not exactly the same. To compensate this residual difference, the length $L_{L}$ and $L_{R}$ are slightly adjusted. 
(a)

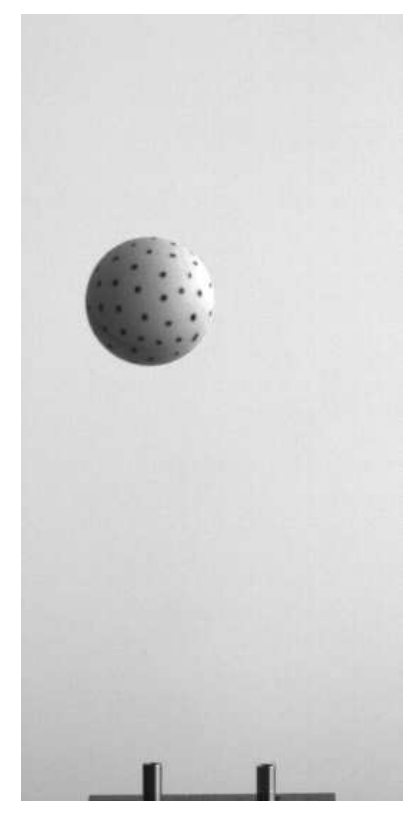

(b)

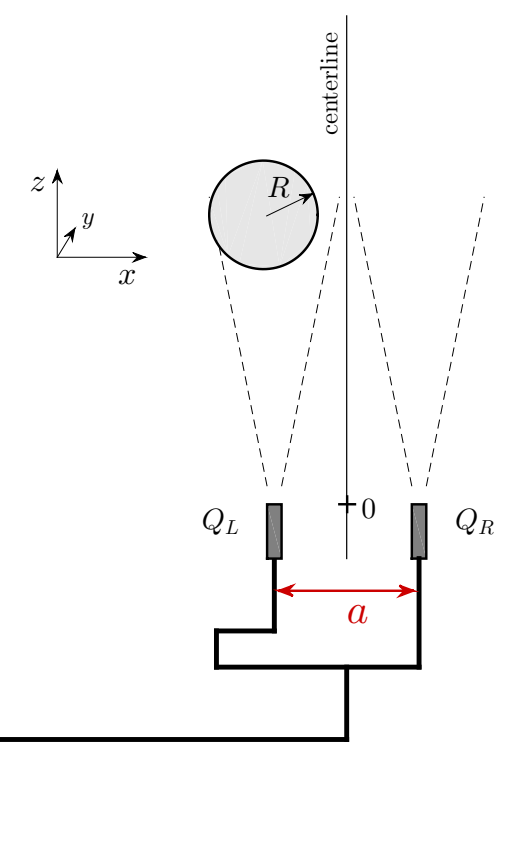

FIG. 1. (a) Photography of a sphere in levitation above two vertical turbulent jets of equal flow rates. The distance between the nozzles is $36.1 \mathrm{~mm}$ and the diameter of the ball is $40 \mathrm{~mm}$. The ball is marked with black dots distributed on its surface. (b) Sketch for the experimental setup. The origin of the frame is at the middle of the two nozzles. The length of the tubes from the T-junction is $L_{L}$ for the left jet and $L_{R}$ for the right jet. $Q_{L}$ and $Q_{R}$ are the flow rates for the individual jets. $a$ is the separation between the nozzles.

The levitation threshold imposes that the momentum flux for one nozzle has to be larger than the weight of the ball. The threshold in velocity is of the order of:

$$
\rho_{a} S_{N} v_{N}^{2}=m g
$$

in which $\rho_{a}$ is the air density, $S_{N}$ the cross section of the nozzle, $v_{N}$ the velocity at the nozzle and $g$ the gravity acceleration. With a ping-pong ball and a tube of $4 \mathrm{~mm}$, one gets $v_{N}=42 \mathrm{~m} / \mathrm{s}$ which corresponds to a Reynolds number at the nozzle $R e \sim 10^{4}$. For all the experiments presented below, the Reynolds number will be larger than $10^{4}$ which means the jet is fully turbulent, with a universal structure scaled by the velocity at the nozzle.

The trajectory of a trapped sphere is recorded with a camera. A white LED screen is positioned behind the sphere in levitation. The location of the sphere in pixels is obtained from the center of mass of the complementary image in normalized units for which 1 is the normalized grayscale index for the sphere and 0 is for the illuminated background. A space calibration is used to establish the relation between pixels and locations in the observation plane $x z$. The calibration compensates the misalignment of the camera and also the radial distortions induced by the camera lenses.

\section{SYMMETRIC TRAP}

In this section, the configuration with two jets of equal flow rate is considered. At low flow rates, close to the levitation threshold, the levitation is above one of the two jets without the influence of the other. The question addressed here is the location and the dynamics of a trapped sphere when the flow rate is increased in a regime for which the levitating sphere is under the influence of both jets. 


\section{A. Transition from a double trap to a single trap}

\section{Trapping map}

A first characterization of the trapping location is realized with a continuous increase of the flow rate during a video acquisition of the order of 5 minutes at a rate of 20 frames per second. The flow rate is varied continuously at the pressure controller between a lower value corresponding to a trapping position as close as possible to the nozzle to an upper value for which the sphere escapes from the trap. The trajectory of the sphere is recorded and the histogram of the particle probability location is computed in the $x z$ plane. The trapping map of the particle in the double jet configuration is presented in figure 2 for a spacing between the two nozzle of (a) $31.9 \mathrm{~mm}$ and (b) $36.2 \mathrm{~mm}$.

(a)

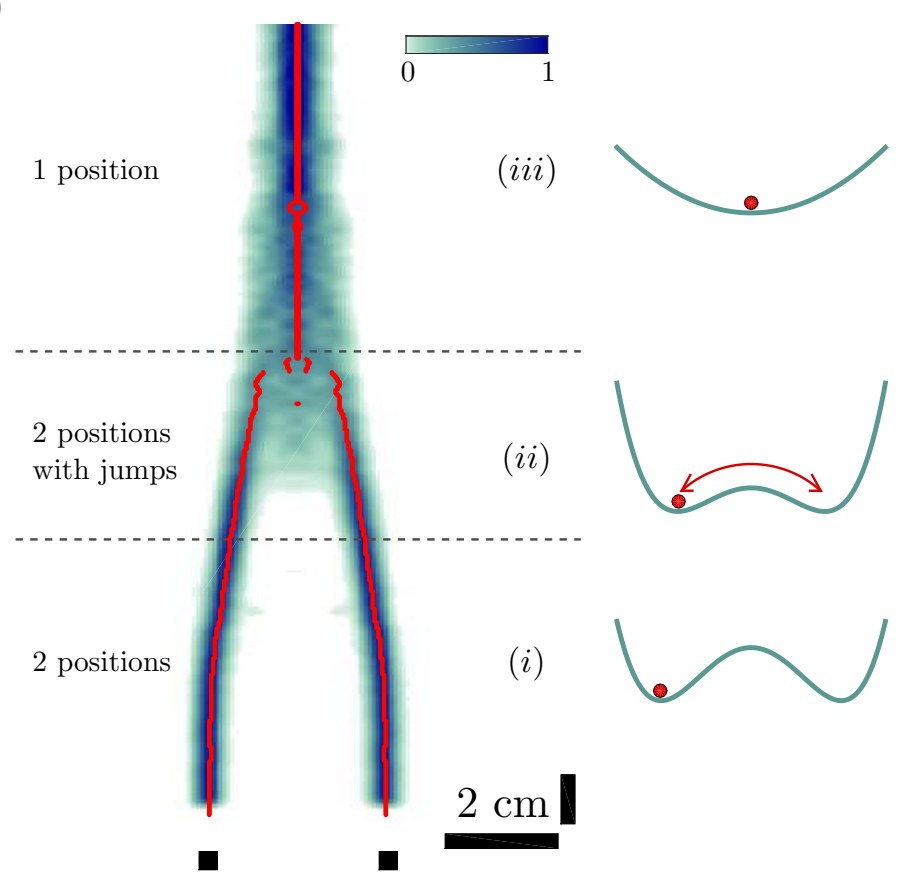

(b)

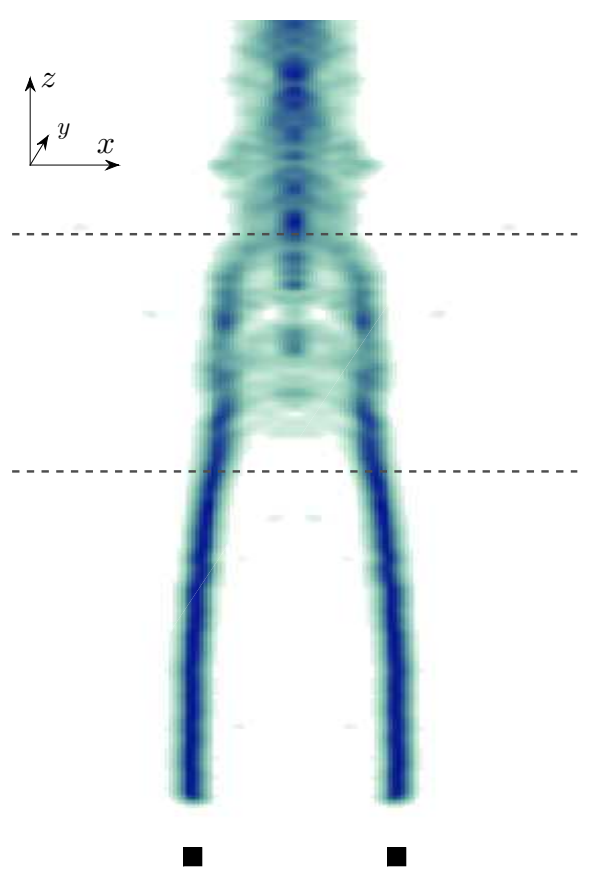

FIG. 2. (a) Probability map of a sphere in levitation above two jets of equal flow rate with separation $a=31.9 \mathrm{~mm}$. The map is obtained by varying the total flow rate and averaging the trajectory of the sphere. The red line indicates the maximum of the probability of presence for each horizontal line of the map. The dashed-lines are guiding lines to identify the three regimes (i) two positions without jumps (ii) two positions with noise-induced switching (iii) central position. The map is represented with a horizontally stretching of a factor 2 . A linear color scale is used with 1 corresponding to the maximum of probability of the map. (b) Probability map of a sphere in levitation with a separation $a=36.2 \mathrm{~mm}$ between the nozzles.

The trapping map is obtained after two steps of signal processing. First, the trajectory is superimposed to its leftright symmetric image. Second, the sum of the pixels of the trapping map is normalized to unity for each horizontal line of the map. The first step ensures a symmetric map without a bias coming from the difference in the time spent in the left branch and the right branch at low flow rates. The second step compensates the non-uniform sampling in $z$.

For the lower flow rates, the ball levitates above one of the two nozzles. As the flow rate increases, the horizontal position of the ball approaches the centerline of the two jets and, above an altitude $z_{c}$, the two levitation branches merge in a single central branch corresponding to a centered levitation. $z_{c}$ corresponds to a critical point between a double-well potential and a single well potential.

For each line of the map, the position of the maximum is identified. A set of red solid lines are plotted on top of the trapping map to show the position of the local maxima. The red lines reveal the backbone of the trapping map. The structure of this backbone is characteristic of a pitchfork bifurcation for a dynamical system ${ }^{26}$.

The fork was observed for a nozzle separation ranging between $28 \mathrm{~mm}$ and $44 \mathrm{~cm}$. Below $28 \mathrm{~mm}$, the ball can be trapped above one of the two jets at low flow rates. For larger flow rates, the transition to a centered levitation is 


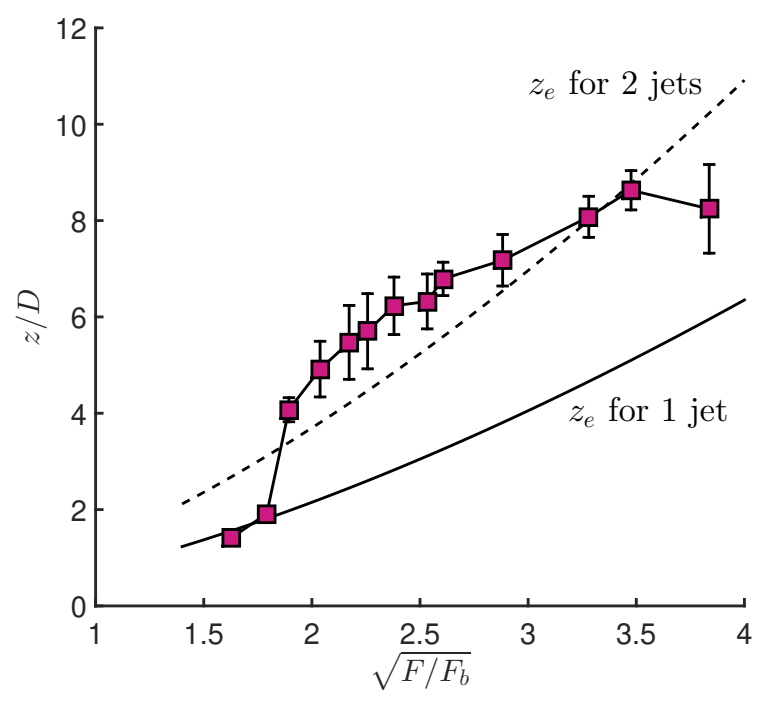

FIG. 3. Altitude $z$ of the levitating sphere with diameter normalization as a function of the square root of the normalized input force per jet. $F_{b}$ is the weight of the sphere. The solid line is the relation for $z$ predicted for a sphere in levitation with a single jet of input force $F$. The dashed line is the same relation with an input force $2 F$. The error bars indicate the vertical RMS displacement of the sphere over time while $F$ is fixed.

not observed because the fluctuations are too strong to trap the particle. Above $44 \mathrm{~mm}$, the centered levitation is too high to be reached and the particle escapes before.

One could notice that the lines in the bifurcation map in figure 2 are not well resolved as one should expect for a conventional pitchfork bifurcation. First, the particle 3D position $X, Y, Z$ is a function of the control parameter, here the flow rate. It means that the map itself is not a straightforward representation of a pitchfork bifurcation. However, we show in the following section ЏIIA2 that the average particle altitude is a monotonic increasing function of the flow rate. Secondly, the particle fluctuations are large, typically of the order of the particle radius even with one jet. In the case of the double jets, the existence of a critical point implies a softening of the confinement. As discussed below, these fluctuations are mainly attributed to the driving by the jet intrinsic fluctuations.

\section{Particle altitude}

A second set of experiments is performed in which the ball's trajectory is measured for a constant flow rate. The flow rate is increased step-by-step and the trajectory of the ball is recorded during acquisitions of 2 to 3 minutes typically for each step at a rate of 60 frames per second. The results for the altitude of the ball are presented in figure 3. The square root of the normalized force $\sqrt{F / F_{b}}$ is presented on the horizontal axis. $2 F$ is the total input force of the two jets and $F_{b}=m g$ is the weight of the ball with $m=2.7 \mathrm{~g}$ and $g$ the gravity acceleration. For each data points, the flow rate is maintained constant and the average altitude $z_{e}$ is measured from the recorded trajectories. The altitude is represented in normalized units $z_{e} / D$ with $D$ the particle's diameter.

The force of the two jets $2 F$ is measured after each acquisition by placing a flexible cantilever plate above the two jets. The cantilever spring constant is measured by positioning a weight on the cantilever and measuring the associated deflection. The cantilever is placed at a distance of $32 \mathrm{~mm}$ above the nozzle and the cantilever is sufficiently large to be fully impacted by the two jets. Because the force of a turbulent jet is preserved $\stackrel{12,13}{ }$, the total force $2 F$ should not depend on the distance of the cantilever to the nozzle and, by symmetry, $F$ is the input force for each individual jet.

The force for one jet measured with the cantilever is $F=c_{D} / 2 \rho S_{N} V_{N}^{2}$ with $\rho$ the air density, $S_{N}$ the cross sectional area of one nozzle and $v_{N}$ the RMS velocity at nozzle. The square root of the normalized force can be written as:

$$
\sqrt{\frac{F}{F_{b}}}=\sqrt{\frac{2 c_{D}}{6}} \mathcal{F}_{r} \mathcal{K}^{-\frac{1}{2}} \mathcal{D}^{-\frac{3}{2}}
$$

in which the dimensionless parameters introduced ${ }^{\underline{6}}$ are: $\mathcal{F}_{r}=v_{N} / \sqrt{D_{N} g}$ the Froude number, $\mathcal{D}=D / D_{N}$ the diameter 
ratio with $D_{N}=4 \mathrm{~mm}$ the nozzle inner diameter and $\mathcal{K}=\rho_{b} / \rho$ the density ratio. According to relation $(2), \sqrt{F / F_{b}}$ is proportional to the exit velocity $v_{N}$. Consequently, $\sqrt{F / F_{b}}$ may also be interpreted as a normalized input flow rate and will be referred as such in the following.

The solid line in figure 3 is an empirical estimation of the equilibrium distance $z_{1}$ as a function of the input force for a single jet:

$$
\frac{z_{1}}{D}=\left(\sqrt{\frac{F}{F_{b}}}\right)^{\alpha}
$$

The value of the exponent $\alpha=1.562$ is obtained after the analysis of the experimental data points reported in a previous work about the levitation in a single jet. The dashed line corresponds to the estimation of the equilibrium distance $z_{2}$ for a single jet but for a double input force:

$$
\frac{z_{2}}{D}=\left(\sqrt{\frac{2 F}{F_{b}}}\right)^{\alpha}
$$

The experimental data in figure 3 follows the master curve for a jet with an input force $F$ at low flow rates and the master curve for a jet with an input force $2 F$ at high flow rates. This result is consistent with the trapping maps presented in figure 2. At low flow rates, the sphere levitates above one of the two jets without the influence of the other. At large flow rates, the two jets merge into an equivalent single jet with a trapping distance consistent the sum of the forces from the two individual jets.

\section{Particle fluctuations}

The set of experimental data for which the flow rate is increased step-by-step is analyzed to extract the fluctuating dynamics of the levitating ball. Figure 4 shows the probability density functions (PDFs) of a ball levitating above two jets for different input flow rates. The normalized flow rate $\sqrt{F / F_{b}}$ is indicated in the upper left corner of each image.

For the lowest flow rates $\left(\sqrt{F / F_{b}}=1.63,1.79\right.$ and 1.89$)$, the sphere explores only one of the two jets. The first 3 maps presented in figure 4 are obtained by superimposing the PDFs obtained with the sphere initially above the left jet and the PDFs with the sphere initially above the right jet.

For the images with flow rates $\sqrt{F / F_{b}}=2.04,2.17,2.26,2.38$ and 2.53 , the PDFs show two symmetric branches associated with the left and right jets. The fluctuations and the proximity of the branches is such that the ball transits spontaneously between the two branches. The switching rate is computed as the ratio of the number of crossings of the vertical centerline divided by the duration of the video acquisition. This switching rate is indicated next to PDFs for the corresponding images. During a switching event, the horizontal fluctuation is correlated to an upward motion. This correlated motion has a signature in the PDFs with a probability pattern resembling an inverted V letter.

At large flow rates $F_{b} / F>2.61$, the PDFs have their maxima on the centerline.

The vertical fluctuation is indicated in figure 3 in which the standard deviation for the vertical motion around the equilibrium position is represented for each data point. The amplitude of the vertical fluctuation is non monotonic and it shows a local maximum in the transition region between $\sqrt{F / F_{b}}=2$ and 2.5 and a local minimum around $\sqrt{F / F_{b}}=3$. The images in figure 4 also show a vertical spread of the PDFs at least twice larger for $\sqrt{F / F_{b}}=2.17$ than for $\sqrt{F / F_{b}}=2.61$

The trajectories $x(t)$ and $z(t)$ of the levitating ball for the probability map $F_{j} / F=2.38$ are presented in figure 15 in the appendix B1. The trajectories and their probability of presence show that the fluctuations in positions are mostly Gaussian which means that the particle fluctuations is dominantly attributed to the driving by the noise. The noise comes from the velocity fluctuations that are of the order of $30 \%$ for a jet in the turbulent regime. Close to the critical point where the left and right branches merge, the fluctuations are enhanced because of the softening of the trap caused by the potential inversion depicted in figure 2.

\section{B. Rotation}

The rotation speed of a particle in levitation is measured by tracking the particle orientation. The white particle is covered with an ensemble of black dots of typical size 1-2 mm and randomly distributed onto the sphere surface with a first neighbor distance of $1 \mathrm{~cm}$ typically. 

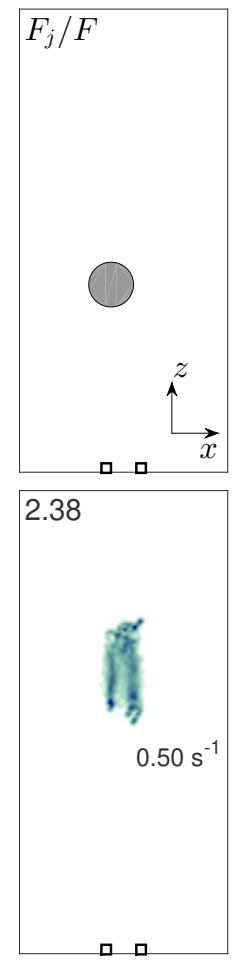

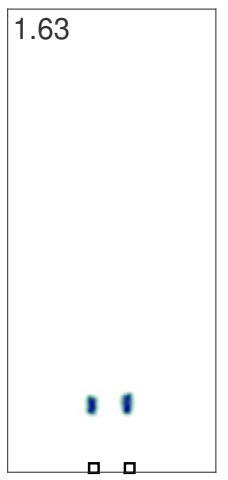

2.53
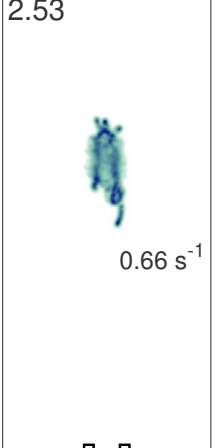

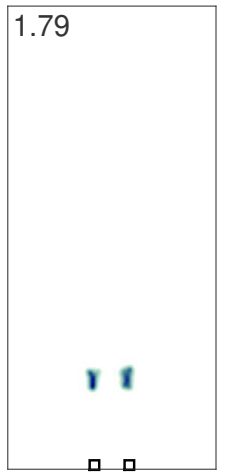

2.61

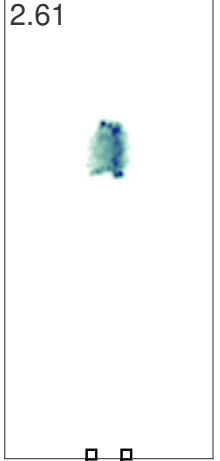

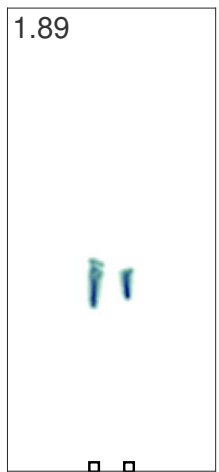

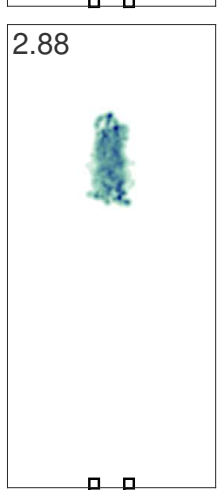

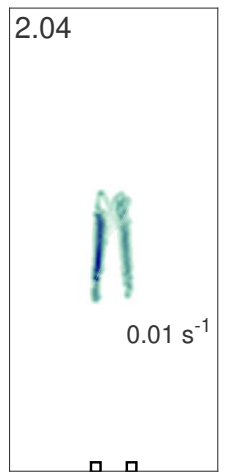

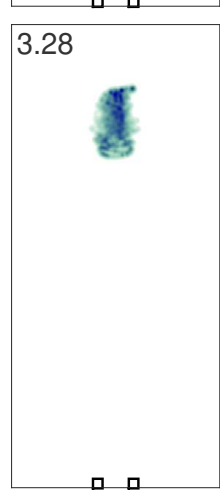

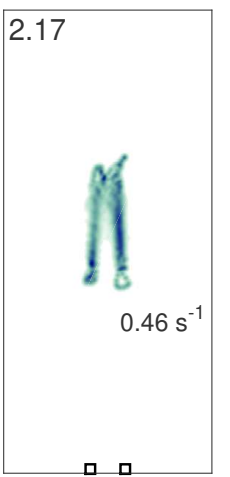

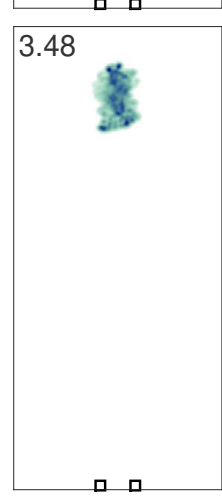

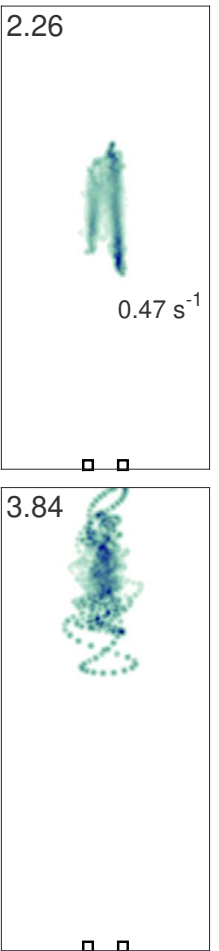

FIG. 4. Probability maps of a levitating sphere with two jets of equal flow rate for different values of the normalized square root of the input force $\sqrt{F / F_{b}}$. This value is indicated in the upper left corner of each map. For intermediate input forces, the sphere is trapped either above the left jet or the right jet and switches between the two branches with a rate $\left(\mathrm{s}^{-1}\right)$ indicated in the images. The first image is a sketch of the experiment with a levitating sphere above the left nozzle.

An acquisition of a levitating particle is performed with a slowly varying flow rate with the same protocol used to obtain the trapping maps in section IIIA 1. Here, the camera acquisition frame rate is 100 frames per second and the spacing between the jets is $a=35.8 \mathrm{~mm}$ which is about the same spacing as for figure 2 (b). Instead of a presence map (PDF), an angular velocity map is constructed. The angular motion of the particle for each time step is inferred from the analysis of two successive images: first, the dots on the particle are tracked in the projected field of view of the camera. Second, the spherical coordinates are used to express the 2D coordinates in pixels onto the particle surface as a 3D vector. This step involves the estimation of the radius in pixels and it gives access to the orientation of the sphere. Finally, an elementary 3D rotation is determined to superimpose the orientation of the sphere at a given frame with the orientation of the sphere for the next frame. This elementary rotation is proportional to the angular velocity of the sphere.

The rotation speed of a levitating particle is presented in figure 5 (a). The angular rotation speed is presented for the 3 components with normalized units with $\widetilde{\omega}_{i}=\omega_{i}, / \omega_{0}, i=\{x, y, z\}$ in which $\omega_{0}$ is the ratio of the typical velocity of the fluid around the ball $v_{s}$ divided by the radius of the ball $R$. The typical velocity around the sphere is approximated to the terminal velocity of the same sphere settling in a fluid at rest $\underline{11}$. For a ping-pong ball in air $v_{s}=8.3 \mathrm{~m} \cdot \mathrm{s}^{-1}$. The normalized angular velocity can be viewed as the ratio of the particle surface velocity to the typical fluid velocity around the particle.

For the lowest flow rates, the particle levitates above one of the jets without the influence of the other. In this regime, there is no significant rotation of the particle for the 3 components.

For the intermediate flow rates, the particle shows a biased rotation speed for $\omega_{x}$ that reaches about $8 \%$ of the typical fluid velocity $v_{s}$ around the particle for a trapping distance of $z_{e}=3.3 D$. This regime is for the particle still levitating above one of the jet but with the influence of the other jet. The rotation is a signature of shear.

In the intermediate regime, there is almost no rotation for the $x$ and $z$ components. This is general to all regimes because the vertical plane containing the nozzles ( $x z$ plane) is a plane of symmetry of the experimental configuration. This means that no rotation component can be contained in this plane, except the rotation due to the fluctuations.

At high flow rates, the particle levitates in an equivalent centered jet without shear on the centerline. As a result, the biased rotation progressively drops to zero for $z_{e} / D \approx 6$. The estimated transition to a central jet is indicated by 
(a)

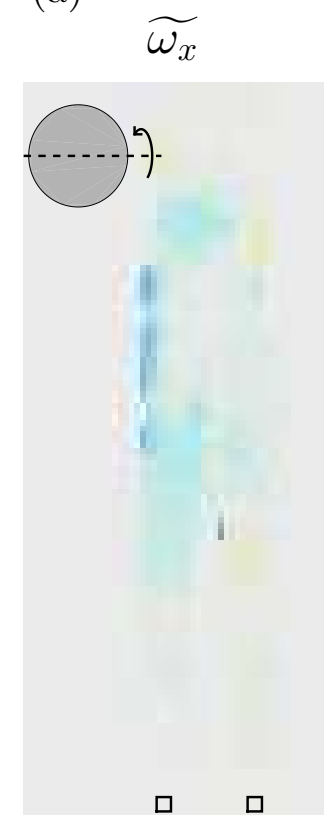

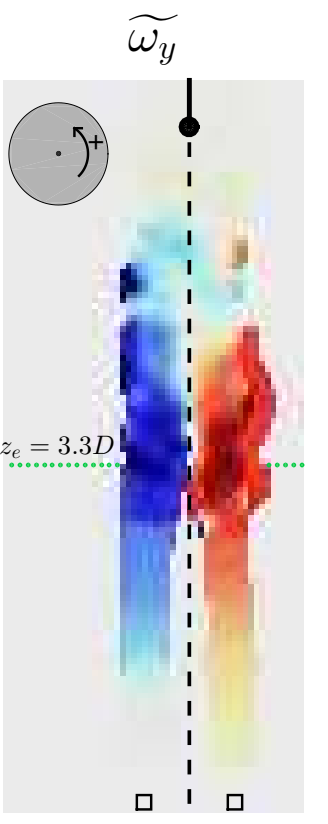

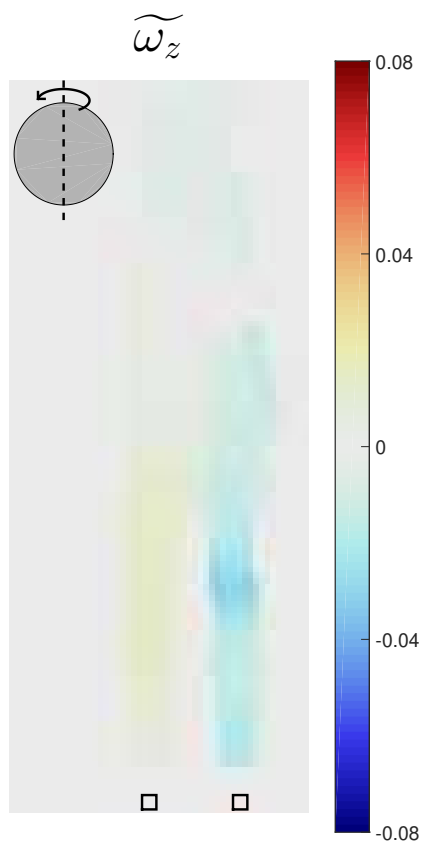

(b)

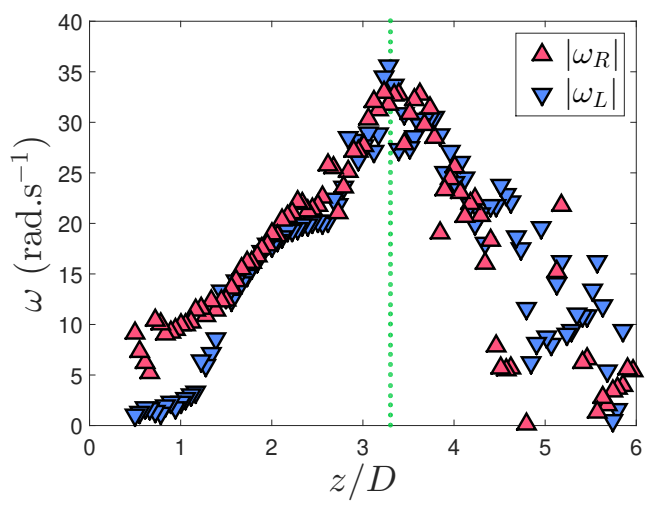

FIG. 5. (a) Angular frequency maps $\widetilde{\omega}_{i}(x, z)$ for a sphere in levitation above two jets of equal flow rates. The map is obtained by varying the jets flow rates and by representing the angular frequency components as a function of the particle location. The ball has a significant angular velocity $\widetilde{\omega_{y}}$ for a rotation perpendicular to the plane $x z$ containing the jets. The ball rotates positively when it is located above the right jet and negatively when it is located above the left jet. The angular velocity is normalized by $v_{s} / R$ in which $v_{s}$ is the settling velocity of the ball and $R$ its radius. The map shows the rotation of the sphere mostly in the double-well region marked the vertical dashed line. Above the altitude $z / D=6$, the levitation is centered and no biased rotation is observed (b) Absolute value of the angular frequencies as a function of the normalized altitude of the sphere for the left branch $\omega_{L}$ and for the right branch $\omega_{R}$. The dashed line indicated the altitude for the maximal rotation at $z / D=3.3$.

a dot separating the centerline from a dashed to a solid line in figure 5 (a). The altitude of this transition is $z_{e}=6.2 D$

Figure 5 (b) shows the rotation speed $\omega_{y}$ as a function of the trapping distance for two regions: the region above the right jet ( $\omega_{R}$, upward triangles) and the region above the left jet $\left(-\omega_{L}\right.$, downward triangles). First, the rotation speeds are almost exactly opposed except close the nozzle. Second, the rotation speed increase quasi-linearly with the distance up to a distance of $z_{e} \approx 3.3 D$ marked by a dashed line in both figures 5 (a) and (b).

The sign of the rotation is positive for the levitation above the right jet and negative for the levitation above the left jet. This rotation sign is counter-intuitive because one could expect that a particle in levitation in the left jet would be driven in positive rotation with the influence of the right jet. The reason for the negative rotation velocity observed is due to the right jet inducing an attractive lateral force that shifts the ball slightly off-center of the left jet axis.

\section{FORCE FIELDS}

As discussed in the introduction, the structure of turbulent jets is universal. In the following, we will assume that the structure for the two jet configuration is also universal. As a consequence, the force field for the symmetric configuration can be established for one value of the input flow rate. 


\section{A. Measurement}

A mapping of the force field with two jets is performed by placing a sphere with $D=40$ mm on a flexible cantilever. The separation between the jets is $32.1 \mathrm{~mm}$ and the flow rate configuration is symmetric.

The cantilever is a flexible cylindrical rod with a diameter of $4 \mathrm{~mm}$ orientated in the $y$ direction perpendicular to the observation field of view. The deflection of the ball in the $x$ and $z$ directions give access to the horizontal $F_{x}$ and vertical $F_{z}$ components of the fluid force acting on the sphere via the spring constant of the cantilever.

The cantilever with the attached ball is placed at different positions in the plane $x z$ to obtain a set of force measurements for $F_{x}$ and $F_{z}$ on a binning grid with a typical mesh size of $10 \mathrm{~mm}$. A spatial Gaussian filtering is used to obtain a map representation of the force fields for the horizontal component in figure 6 (a) and the axial component in figure 6 (b).

(a)

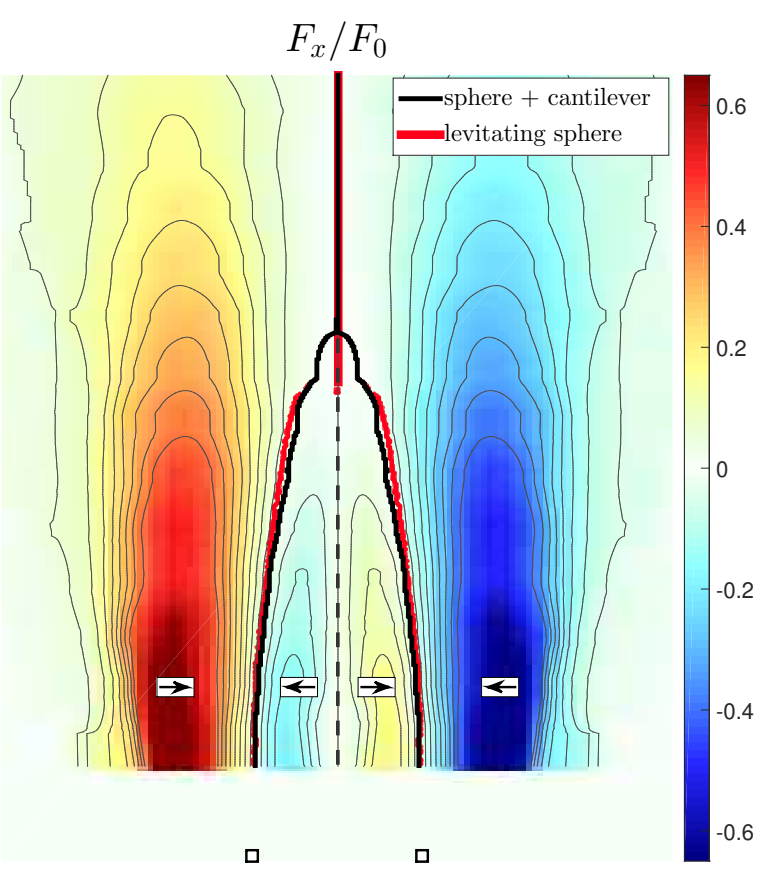

(b)

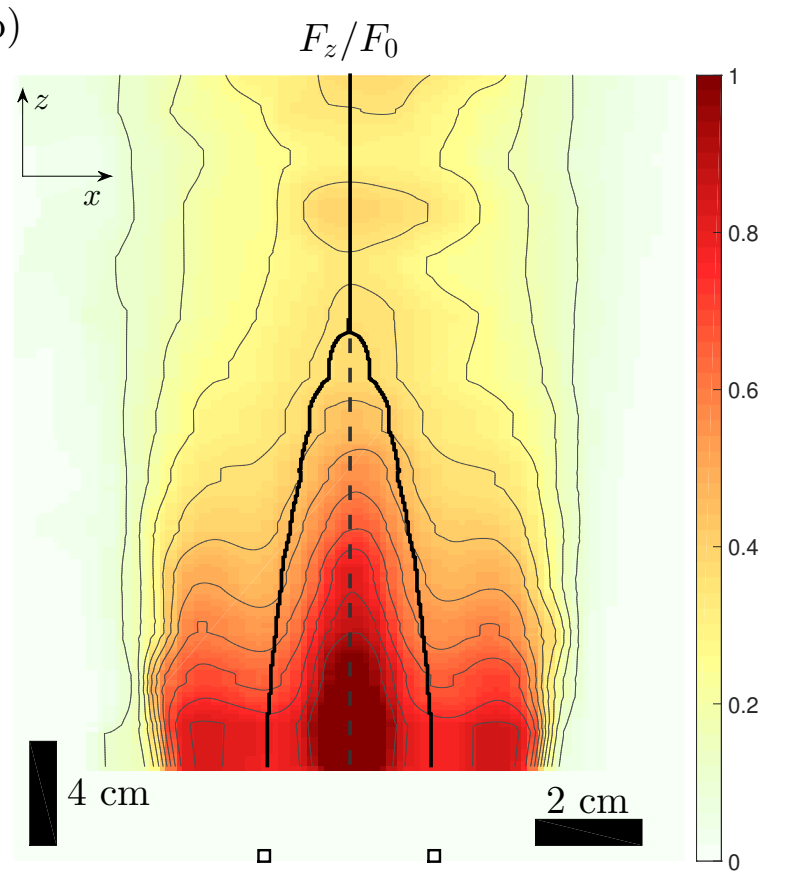

FIG. 6. (a) Horizontal force map for a fixed sphere above two jets of equal flow rates. The ball is fixed on a flexible cantilever and positioned at different location to map the force field. The thin solid line marks a stable horizontal equilibrium $F_{x}=0$, $\partial F_{x} / \partial x<0$. The dashed line on the centerline is an unstable equilibrium line. The thicker solid line is the probability map backbone represented in figure 2 (a) for the experimental results for the same nozzle separation. (b) Vertical force map. The forces are in normalized units.

The first force map $F_{x}$ in figure 6 (a) is used to identify the equilibrium lines corresponding to $F_{x}(x, z)=0$ in black solid line. The term equilibrium lines refers here to the ensemble of position occupied by the sphere in levitation when the flow rate is varied and with an averaging of the fluctuations. Four branches are found. First, there is a pair of two symmetric branches starting from the left and right jets. Those 2 branches are stable as indicated by the arrows showing the orientation of the force $F_{x}$ in each region. Second, the central branch in the upper part of the map indicates the region where the two jets merge into a central jet. This branch is also stable. The last branch is the central branch marked by a dashed-line. This line corresponds to an unstable equilibrium point.

Another stability line is presented in the same figure with a red thick solid line. This stability line is the bifurcation fork presented in figure 2 for the same nozzle configuration. Although the two sets of stability lines are very close, one could see some differences. First, the merging point is slightly lower for the free levitating sphere. It is possible that the large fluctuations are playing a role in the trapping map for a levitating particle. In general, it is known that fluctuations can shift the position of a sharp transition by smoothing the force landscape around the transition point. Here, a smoothing of the force map $F_{x}$ by a Gaussian kernel results in a downward shift of the merging point. A second difference is the fact that the stability fork for the levitating sphere remains a bit larger in the lower part 
of the force mapping. This slight shift could be attributed to the fact that the levitating sphere is free to rotate and indeed spins significantly as discussed in section $\amalg$ W This rotation may involve a lateral force due to Magnus effect.

The second force map $F_{z}$ in figure 6 (b) shows the axial component of the force. This force is aligned with the weight of the ball. The thick solid and the dashed lines represents the same set of lines as in figure 6 (a). For the two maps, the thinnest lines are isoforce lines. The position of a sphere in levitation corresponds to the intersection of the stability fork established with $F_{x}=0$ and the isoforce line $F_{z}$ that equals the weight of the levitating particle.

The vertical force $F_{z}$ appears to decrease with the altitude when the stability lines are followed, at least in the lower half of the mapping. If one assumes that the force profile is proportional to the input force of the two jets $F$, the monotonic decrease means that the average altitude of a levitating particle is continuously increasing with an increasing flow rate. It is not clear if the vertical force is strictly monotonic above the merging point. The particle fluctuations in figure 4 indeed show large vertical fluctuations at $\sqrt{F / F_{b}}=3.31$ which may be attributed to a region with multiple stable points.

It is interesting to notice that, below the merging point, the trapping position at a given altitude is not where $F_{z}$ is maximal. It appears that $F_{z}$ is always maximal on the centerline, even if this equilibrium line is not stable. A similar observation was reported for the levitation in a single turbulent jet 27 at moderate distance $z=3.5 D$ for which the vertical component of the force is not maximal at the trapping position.

Another important point is the softening of the trap at the merging of the branches. For the horizontal restoring force, there is a rigorous cancellation of the equivalent spring force because of the potential inversion. For the vertical restoring force, one can see that the isoforces lines for $F_{z}$ in figure 6 (b) follow the line $F_{x}=0$ close to the inversion point. This means that the softening occurs for both the horizontal and the vertical motion. This is consistent with the increase of vertical fluctuations observed in figure 3 near the transition from two jets to one jet.

\section{B. Velocity and force summation rules}

The object of this section is to propose a prediction for the force field for a sphere with two jets. If the sphere is small, a model for the summation of the velocity is sufficient to predict the force profile. If the sphere diameter is comparable to the jet size, an empirical formulation of the force field for one jet is necessary.

\section{1. velocity field}

There is no superposition principle for the velocity of a fluid in general simply because the governing Navier-Stokes equation is nonlinear. However, an empirical theory for turbulent jets was proposed by Reichardt $\underline{28}$ in which the squared velocity satisfies a linear equation 29 similar to the diffusion equation:

$$
\frac{\partial v^{2}}{\partial z}=\frac{2}{\sigma} z \Delta v^{2}
$$

in which $v$ is the mean velocity for the axial component along $z, \sigma$ a dimensionless parameter and $\Delta v=\partial_{x x} v+\partial_{y y} v$ the two-dimensional Laplace operator. The point $z=0$ is the point source of the jet, also called virtual origin of the jet, generally close to the location of the nozzle in a practical situation.

The mixing process described by equation (5) is similar to a two-dimensional diffusion for $v^{2}$ in the plane $x y$ where $z$ would play the role of time. The effective diffusion coefficient $D=2 z / \sigma$ is proportional to the distance to the source point $z$. The physical origin of this effective diffusion is the mixing induced by turbulence: because the scale of the turbulent fluctuations is increasing proportionally with the distance to the jet origin, the effective diffusion coefficient should increase with the distance to the jet origin. In this perspective, the Reichardt interpretation could be related to Prandtl's mixing length approach in which the mixing length scales proportionally with the distance $z$ to the source point.

Another formulation ${ }^{30}$ for equation (5) is possible with the variable $\mathcal{T}=z^{2}$. In this case, the squared velocity satisfies a diffusion equation with diffusion constant independent of $z$ :

$$
\frac{\partial v^{2}}{\partial \mathcal{T}}=\frac{1}{\sigma} \Delta v^{2}
$$

Equation (6) is exactly a 2D-diffusion equation for the quantity $v^{2}$. The solutions are

$$
v^{2}=\mathcal{P} \frac{2 \sigma}{\pi z^{2}} \exp \left(-2 \sigma \frac{r^{2}}{z^{2}}\right)
$$


in which $\rho \mathcal{P}=\rho \int_{x, y} v^{2} r d r=F_{N}$ is independent of $z$ and corresponds to the input force of the jet discussed in section III A 2 .

Since equation (6) is linear, the superposition principle applies for two or many point sources with non buoyant jets $31-33$ or even, more recently, buoyant jets 34,35 if they are located at the same altitude $z=0$. With two point sources located in $x_{L}=-a / 2$ and $x_{R}=a / 2$, the resulting flow is

$$
v_{z}(x, y, z)^{2}=\frac{2 \sigma}{\pi z^{2}}\left[\mathcal{P}_{L} \exp \left(-2 \sigma \frac{(x-a)^{2}+y^{2}}{z^{2}}\right)+\mathcal{P}_{R} \exp \left(-2 \sigma \frac{(x+a)^{2}+y^{2}}{z^{2}}\right)\right]
$$

in which $\rho \mathcal{P}_{L}$ and $\rho \mathcal{P}_{R}$ are the axial momentum fluxes, $i$. $e$. the forces, for the left and right jets respectively.

\section{Force field for a small sphere}

The validity of equation (8) is tested by measuring the vertical force exerted on a small sphere positioned in the flow of the two jets. For a small sphere, the vertical force is simply proportional to the squared velocity with $F_{l o c}=\left(c_{D} / 2\right) \times \rho \pi r_{0}^{2} v_{z}(x, y, z)^{2}$ in which $r_{0}$ is the radius of the sphere. Here, $r_{0}=3$ mm which is reasonably smaller than the typical scale of the spatial variation of the velocity. The small sphere is pinned onto a needle that is glued to a flat flexible plate that measures the vertical component of the force. The deflection of the sphere is measured as the position of the sphere is varied along the cross flow direction $x$. The separation between the nozzles is $a=40$ mm.

Figure 7 shows the force profile $F_{z} \propto v_{z}(x, y, z)^{2}$ measured for a small sphere for the altitude $z=135 \mathrm{~mm}$ (a) and $z=208 \mathrm{~mm}$ (b). Two other profiles not presented in the figure are measured at $z=156 \mathrm{~mm}$ and $z=183 \mathrm{~mm}$. The scatter points are for the measured force in normalized units in which only the symmetric component of the signal is presented. The asymmetric component is a small contribution of the order of $10 \%$ attributed to uncontrolled parameters such as the non-symmetry of the input flow rates or a misalignment of the cantilever with the observation plane.

The measured force shows a remarkable agreement with the model predicted by equation (8) with a Gaussian profile $\exp \left(-2\left(x \pm a_{f} / 2\right)^{2} / \mathcal{W}^{2}\right)$ for each jet and a sum of the squared velocity. For comparison, a model for the force resulting from a summation of the linear velocity is presented by a dashed line. The adjustment of the two models clearly shows that the linear superimposition applies for the squared velocities and not for the linear velocities.

The two adjustable parameters of the model are the width $\mathcal{W}=z^{2} / \sqrt{\sigma}$ of an individual Gaussian profile and the separation $a_{f}$ between the jets. The width $\mathcal{W}$ of the Gaussian profiles is plotted in figure 7 (d) as a function of the distance to the nozzle. $\mathcal{W}$ is linear with $z$ with a positive vertical shift of $\mathcal{W}=1.6 \mathrm{~mm}$ at $z=0$ which is of the order of the radius of the sphere. Accordingly with Eq. (8), $\mathcal{W}=z / \sqrt{\sigma}$. With the linear fit in figure 7 (d), $\sigma=83$ which is consistent with the value of $\sigma$ for turbulent round jets.

The separation $a_{f}$ between the two jets source points is adjusted for the 4 distances $z=135,156,183$ and 208 mm. The values found for $a_{f}=34.4,37.8,37.6$, and $36.2 \mathrm{~mm}$ are systematically slightly smaller than the separation $a=40$ $\mathrm{mm}$ between the nozzles. This result suggests that there is no rigorous superposition of the squared velocity but a superposition combined with a small attraction of the two jets.

\section{Force field for a large sphere}

If the sphere is large compared to the width of the jet or even of the same order, there is no proportionality between the squared velocity and the force. An empirical formulation for the force acting on a large sphere is proposed in the appendix A for a single jet. Using the same force summation rule as in section IV B 1, one can estimate the force field for two turbulent jets as the sum of the forces from each individual jet. Figure 8 shows the forces $F_{x}$ and $F_{z}$ predicted by the linear summation of the force.

\section{ASYMMETRIC TRAP}

In this section, the levitation dynamics of the ball is investigated when the input flow rate is not equal in the right jet and in the left jet. 
(a)

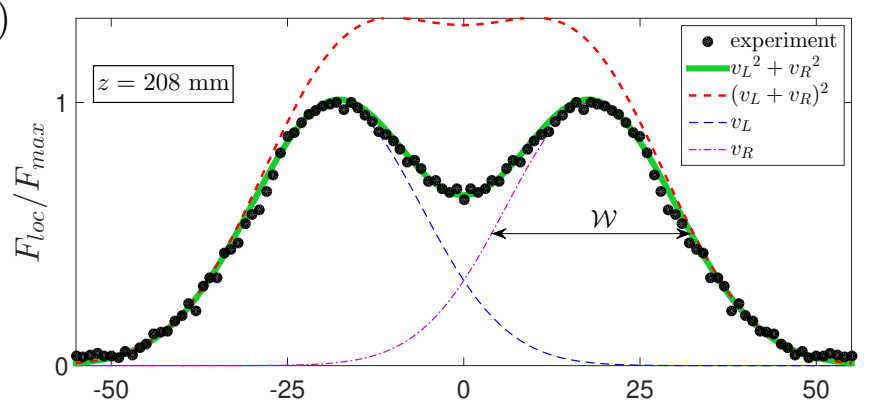

(c)

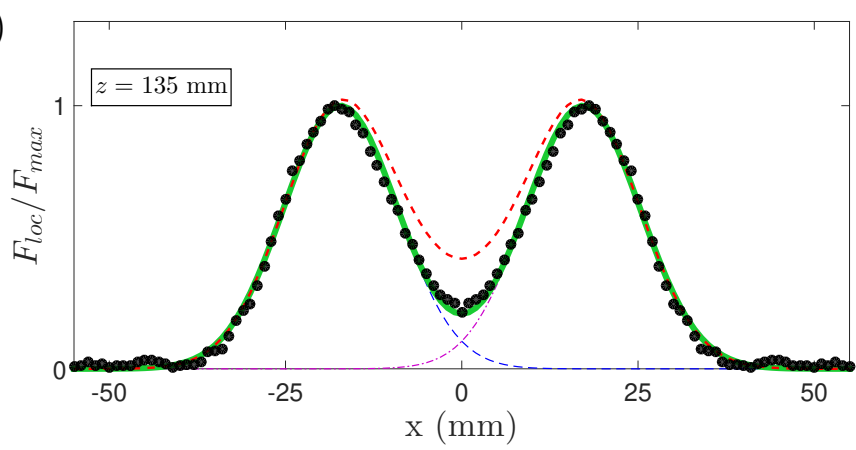

(b)

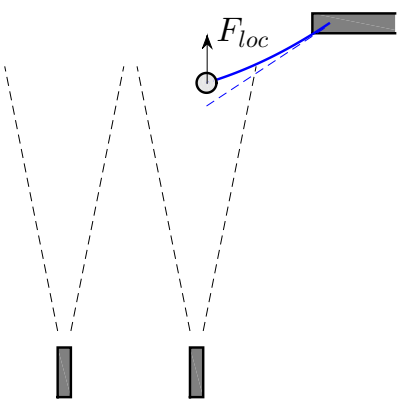

(d)

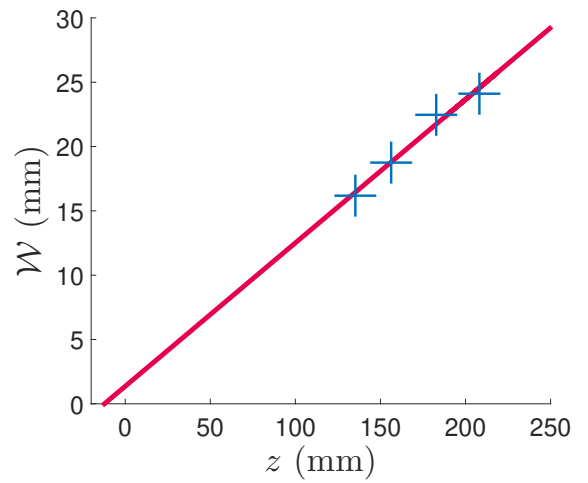

FIG. 7. (a) Normalized force exerted by two jets on a sphere of diameter $6 \mathrm{~mm}$ as a function of the horizontal position. The vertical distance to the nozzles is $208 \mathrm{~mm}$. The thick solid line is the model in Eq. (8) with force summation of the Gaussian profiles $v_{L}^{2}$ and $v_{R}^{2}$ for the individual jets. The free parameters of the model are the separation $d=34.4 \mathrm{~mm}$ between the two Gaussian profiles and the width $\mathcal{W}=14.2 \mathrm{~mm}$ of the profile. For comparison, the force obtained with a summation of the velocities $v_{L}+v_{R}$ is represented by a red dashed line. The nozzles are separated by $a=40 \mathrm{~mm}$. (b) Sketch of the experiment. A sphere of diameter $6 \mathrm{~mm}$ is mounted on a cantilever and positioned in the plane of the two jets of equal flow rates. The sphere is moved step by step horizontally to obtain the force profiles. (c) Normalized force profile at a distance of 135 mm. $d=36.2 \mathrm{~mm}, \mathcal{W}=9.54 \mathrm{~mm}$. (d) Gaussian profile width as a function of the vertical distance to the nozzle. The data are adjusted with a linear relation $\mathcal{W}=p\left(z-z_{0}\right)$ with $p=0.112$ and $z_{0}=-12.2 \mathrm{~mm}$.

\section{A. Trapping map}

An asymmetric flow rate is obtained by increasing the length of the left branch $L_{L}>L_{R}$. This strategy is simple to implement but its counterpart is that the ratio of the left $Q_{L}$ and right $Q_{R}$ flow rates is not constant when the input pressure at the controller is varied. The length for the right branch is $L_{R}=62 \mathrm{~cm}$. Figure 9 shows the trapping map obtained with (a) $L_{L}=78.4 \mathrm{~cm}$ and (b) $L_{L}=92.8 \mathrm{~cm}$.

For the larger length mismatch $L_{L} / L_{R}=1.49$, there is a clear separation of the trapping branches with a short branch above the left jet with the lowest flow rate and a dominating right branch connected to the right nozzle. At large flow rates, the trapping branch is not centered but shifted towards the right jet.

The trapping map was obtained by putting the ball first in the left jet and sweeping up and down the pressure at the controller. During the acquisition, the ball has suddenly jumped from the left branch to the right branch. After this jump, the ball is in stable levitation in the right jet and for a higher altitude than before the jump. When the pressure was swept back to a low value, an hysteresis situation is present and the ball kept levitating in the right branch.

For the smaller length mismatch $L_{L} / L_{R}=1.26$, there is still an asymmetry of the trapping map but the separation between the two branches is small notably because of the position fluctuations of the sphere. This configuration $L_{L} / L_{R}=1.26$ is about the limit where the ball can jump back from the right branch to the left branch thanks to the fluctuations: when the flow rate was slowly swept up, the ball that was initially in the left branch made a first jump to the right branch, then a second jump back to the left branch and a last jump to the right branch, all within less than 5 seconds. 
(a)

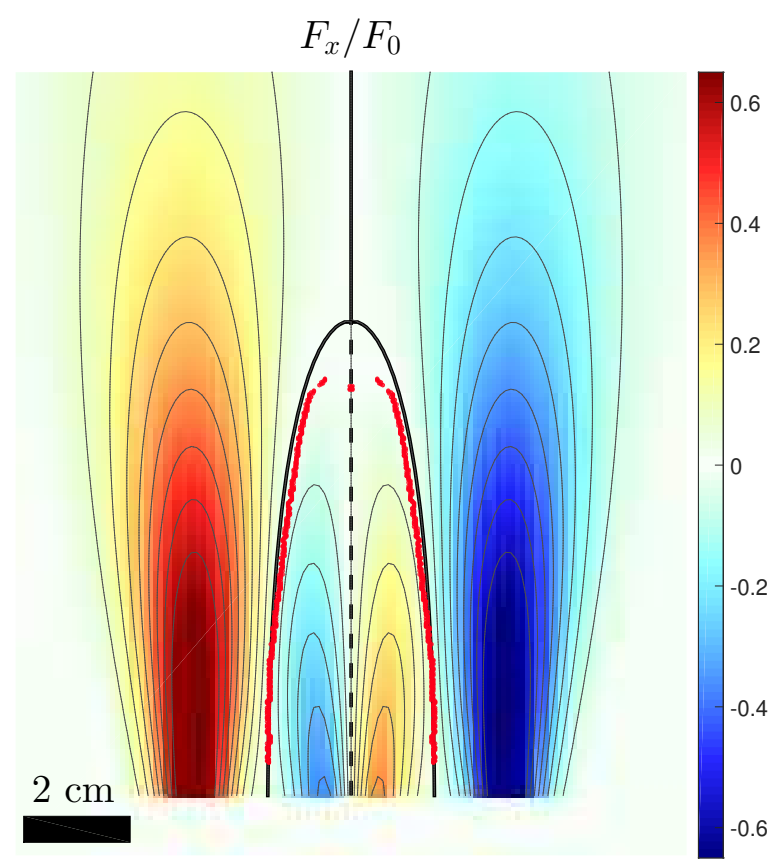

(b)

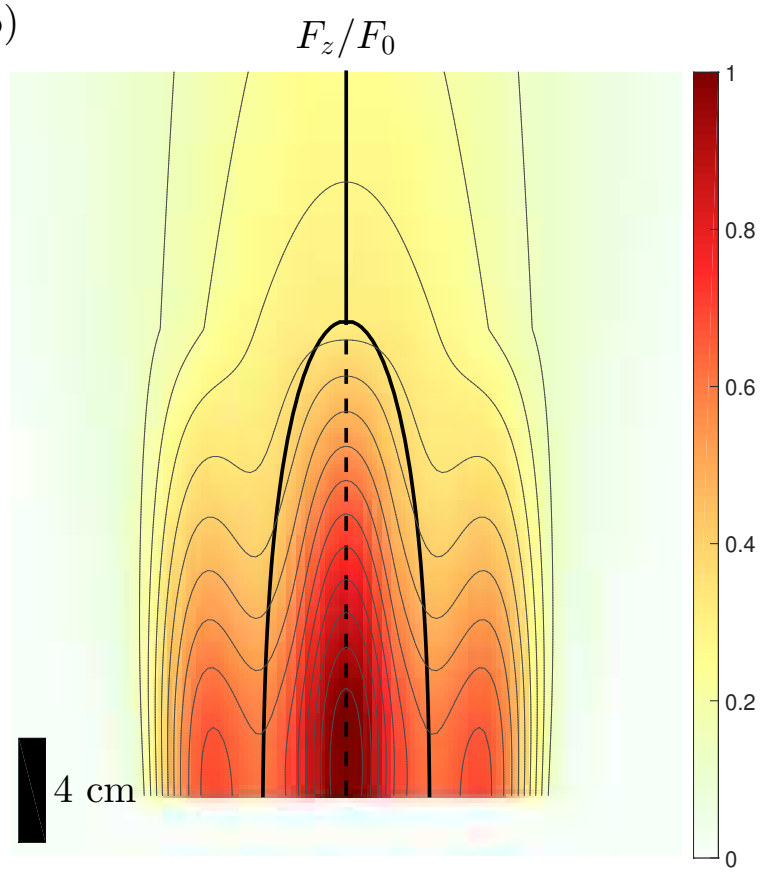

FIG. 8. (a) Prediction of the horizontal force map using the force summation with the force field for one jet established in appendix A The solid lines are the stable equilibrium lines. The dashed line is unstable. The thicker red solid line is the probability map backbone represented in figure 2 (a). (b) Prediction of the vertical force map. The forces are in normalized units.

\section{B. Topology of the trapping lines}

In the case of symmetric flow rates, the trapping maps define a symmetric pitchfork bifurcation structure. For the asymmetric flow rates, the geometry of the trapping line can be obtained using the summation rule described previously to predict the force field when the left and right flow rates are not identical. Figure 10 (a) presents the horizontal component of the force acting on a sphere predicted for a ratio for the jets forces $\mathcal{P}_{L} / \mathcal{P}_{R}=0.9$.

The lines presented in the figure corresponds to the equilibrium lines with $F_{x}(x, z)=0$. The solid lines are stable and the dashed line is unstable. The line structure for asymmetric flow rates is not topologically equivalent to the lines for the symmetric case. The line for the lower flow rate reaches a inflexion point marked by a diamond symbol in the figure. This inflexion point is at the origin of the irreversible jump observed in the experiments.

Figures 10 (b), (c) and (d) represent the potential energies for the horizontal motion as a function of $X$ for the altitudes $z / D=3,4.3$ and 5.3. The potential energy profiles are obtained by integrating the force field $F_{x}$ along the horizontal axis $x$ for each altitude. For $z / D=3$, the energy landscape is a double-well potential, deeper in the stronger right jet. $z / D=4.3$ is a critical altitude with two equilibrium positions, one stable marked by a circle and one semi-stable marked by a diamond symbol. Semi-stable means here that the curvature of the potential is positive at the left (stable) and negative at the right (unstable). The semi-stable point can be viewed as the merging of the two equilibrium points, one stable and one unstable, at the left for $z / D=3$. For $z / D=5.3$, the left equilibrium positions vanishes and the stable position is shifted from the centerline.

\section{PARTICLE DYNAMICS SIMULATION}

In this section, we use the force field prediction established in section IV to compute the dynamics of a particle in levitation. 
(a)

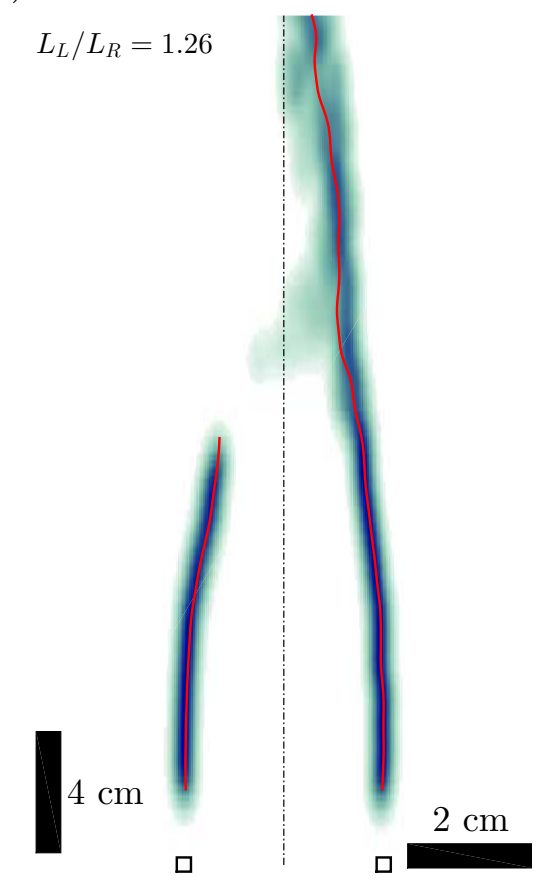

(b)

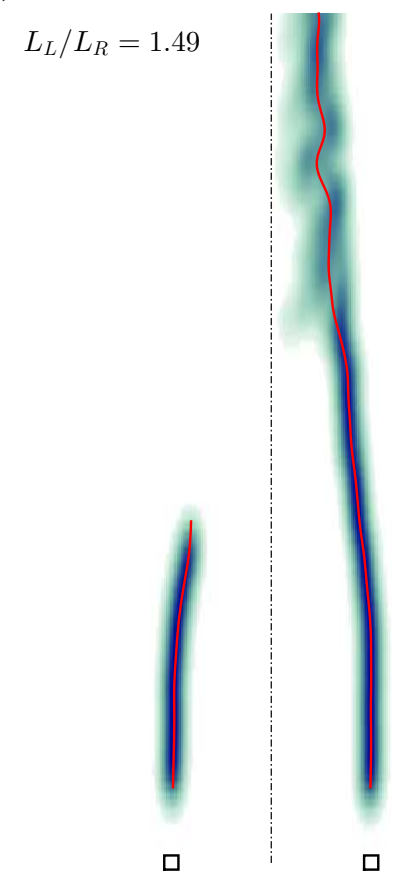

FIG. 9. (a) Probability map of a sphere levitating above two jets of different flow rates. The two jets are connected to a T-junction upstream. $L_{L}=78.4 \mathrm{~cm}$ is the distance between the nozzle and the T-junction for the left jet and $L_{R}=62 \mathrm{~cm}$ is the distance for the right jet. $L_{L}$ is larger than $L_{R}$ which means that the flow rate of the left jet is smaller than the flow rate of the right jet. (b) Probability map for a larger length mismatch $L_{L}=92.8 \mathrm{~mm}$ and $L_{R}=62 \mathrm{~mm}$. Nozzle separation $a=32.4$ mm.

\section{A. Equation of motion}

The equation of motion for a particle in a force field is given by:

$$
\begin{gathered}
\ddot{x}+\alpha_{x} \dot{x}+\frac{F_{x}(x, y, z)}{m}=\eta_{x}(t) \\
\ddot{y}+\alpha_{y} \dot{y}+\frac{F_{y}(x, y, z)}{m}=\eta_{y}(t) \\
\ddot{z}+\alpha_{z} \dot{z}+\frac{F_{z}(x, y, z)}{m}=\eta_{z}(t)
\end{gathered}
$$

in which $\alpha_{i}$ is a set of damping rates, $F_{i}$ the force field established in section IV] $\eta_{i}(t)$ accounts for the noise and $m$ is the mass of the particle.

A relation between the linear damping coefficients $\alpha_{i}$ can be established using a linear expansion of the drag for a small particle with velocity $v_{x}, v_{y}, v_{z}$. The relative velocity of the particle to the fluid is:

$$
\vec{v}=\left(\begin{array}{c}
v_{x} \\
v_{y} \\
v_{z}-V
\end{array}\right)
$$

in which $V$ is the vertical velocity of the fluid. The drag on the particle is:

$$
\overrightarrow{F_{d}}=-\frac{1}{2} c_{D} \rho S v \vec{v}
$$

in which $c_{D}$ is the drag coefficient, $S$ the cross section of the particle and $\rho$ the fluid density. After a Taylor expansion $v_{i} \ll V$, one gets

$$
\vec{F}_{d} \approx-\frac{1}{2} c_{D} \rho S\left(\begin{array}{c}
V v_{x} \\
V v_{y} \\
V^{2}+2 V v_{z}
\end{array}\right)=\left(\begin{array}{c}
0 \\
0 \\
F_{z}
\end{array}\right)+\left(\begin{array}{c}
\alpha_{x} v_{x} \\
\alpha_{y} v_{y} \\
\alpha_{z} v_{z}
\end{array}\right)
$$


(a)

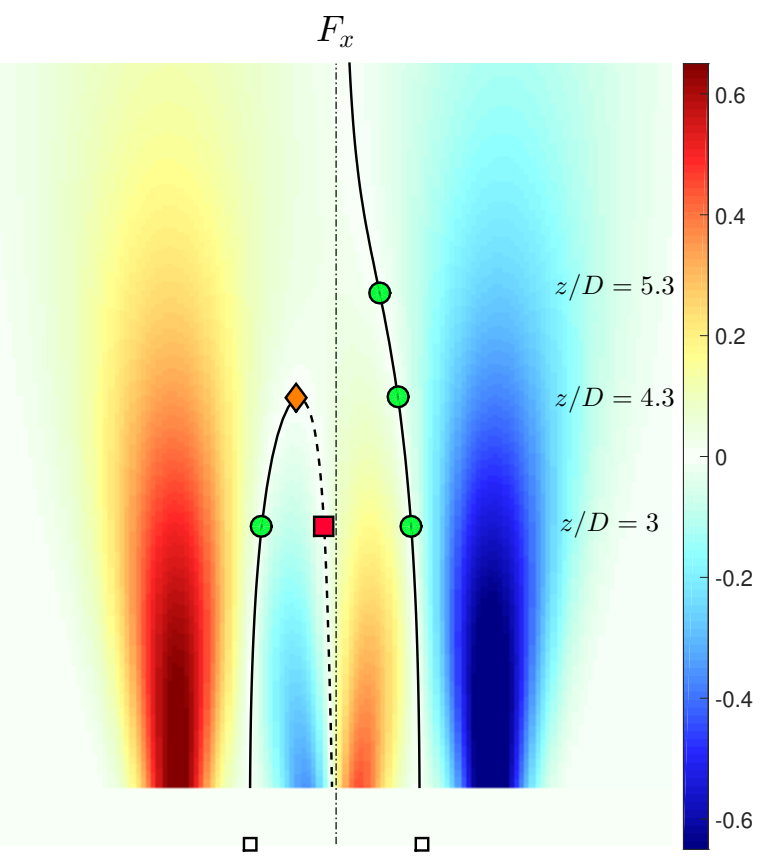

(b)

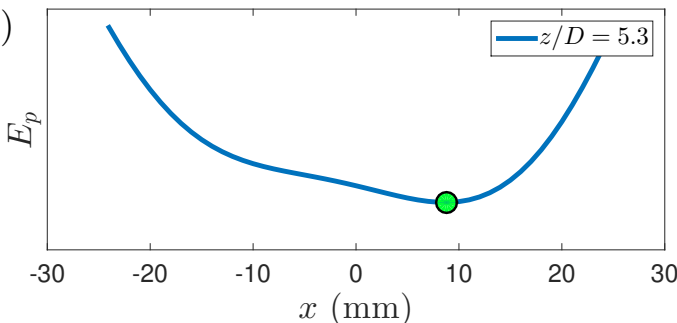

(c)

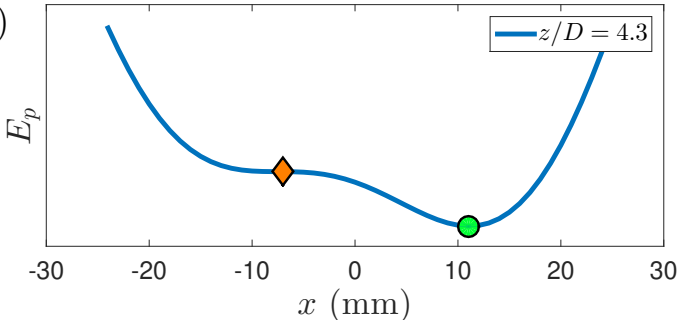

(d)

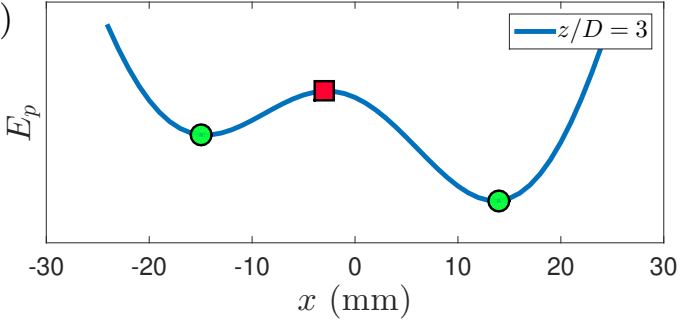

FIG. 10. (a) Prediction of the horizontal force map for two jets separated of a distance $a=x$ mm with different flow rates. The flow rate of the left jet is smaller with a ratio for the jets forces $\mathcal{P}_{L} / \mathcal{P}_{R}=0.9$. The solid line is a stable equilibrium line, the dashed line is unstable. Distance between the nozzles $a=32.4 \mathrm{~mm}$. A thin dot-dashed line indicates the centerline. (b) Energy landscape along a line $z / D=5.3$. There is one stable equilibrium point shifted towards the right jet of higher flow rate. (c) Energy landscape at $z / D=4.3$. The diamond symbol is a hemi-stable point corresponding to the junction of the stable and unstable lines. (d) Energy landscape at $z / D=3$ with an asymmetric double-well potential with two stable points and one unstable point.

with the relations

$$
\begin{aligned}
\alpha_{x}=\alpha_{y} & =\frac{1}{2} c_{D} \rho S V \\
\alpha_{z} & =c_{D} \rho S V
\end{aligned}
$$

A calibration of the vertical damping coefficient is presented in appendix B. The value used in the simulation of Eqs. (91), (10) and (11) are $\alpha_{z}=0.4 \mathrm{~s}^{-1}$ and $\alpha_{x}=\alpha_{y}=0.2 \mathrm{~s}^{-1}$.

The driving noise $\eta_{i}(t)$ models the forcing by the turbulent fluctuations. The noise is zero on average and we assume that the 3 components of the noise are independent and of equal magnitude. We also assume a noise of constant spectral density. The noise satisfies the relations:

$$
\begin{array}{ll}
<\eta_{i}(t)>=0 & \\
<\eta_{i}(t) \eta_{j}\left(t_{0}\right)>=\delta\left(t-t_{0}\right) \eta^{2} & \text { if } i=j \\
<\eta_{i}(t) \eta_{j}\left(t_{0}\right)>=0 & \text { if } i \neq j
\end{array}
$$

in which $i=x, y, z$ and $\eta$ is the magnitude of the noise. The value used in the simulation is $\eta=5.8 \times 10^{-2} \mathrm{~m} \cdot \mathrm{s}^{-2} / \sqrt{\mathrm{Hz}}$ and the calibration of this value is presented in appendix B.

\section{B. Particle dynamics simulation in the symmetric configuration}

The equations of motion for a sphere are solved numerically with two jets with equal flow rates and separated by a distance of $32 \mathrm{~mm}$. The force of the jets is increased step by step with a force per jet that varies between $F / F_{b}=4$ and $F / F_{b}=12$ by steps of 0.2 . This force range corresponds to a vertical distance to the nozzles between $2 D$ and 
(a)

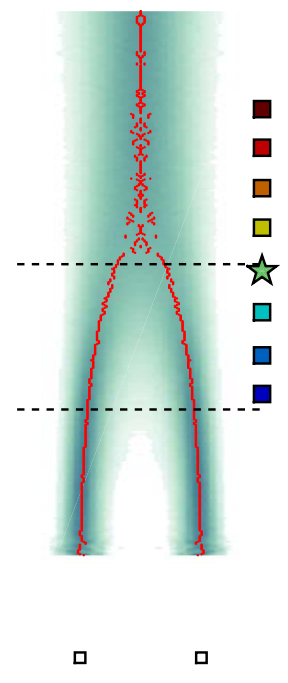

(b)

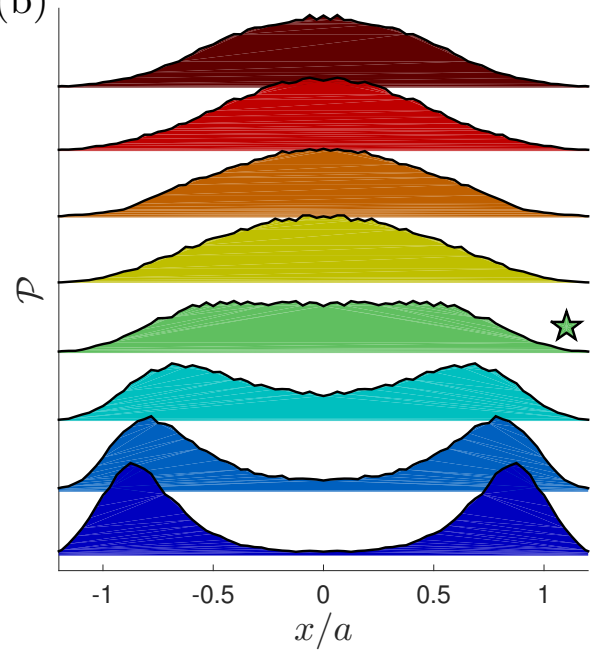

(c)

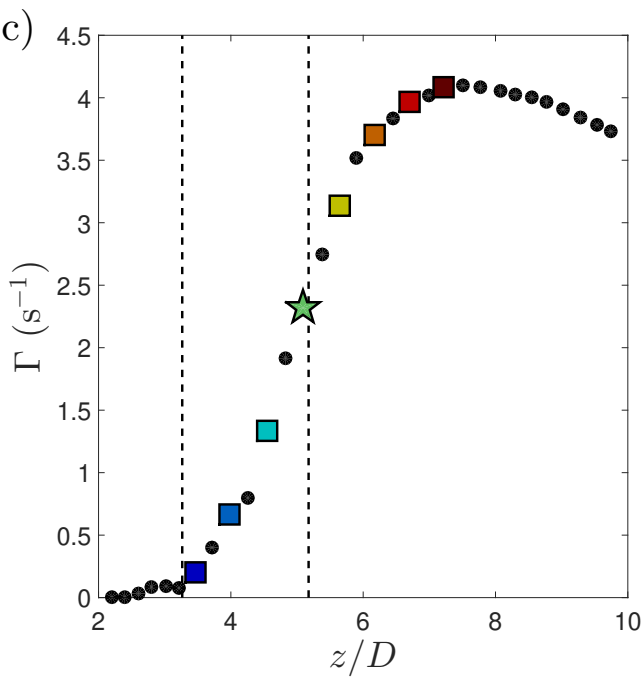

FIG. 11. (a) Probability map for a simulation of a particle in levitation above two jets of equal flow rates (Eqs. (9), (10) and (11)). Nozzle separation $a=32 \mathrm{~mm}$. The dashed lines are the same lines as in figure 2 (a). (b) PDFs for 8 altitudes for the total input force $F / F_{b}=5.4,5.8,6.2,6.6,7.0,7.4,7.8$ and 8.2. The profile for the input force $F / F_{b}=6.2$ is marked with a star symbol and corresponds to a flat probability of presence around $x / a=0$. (c) Transition rate $\Gamma$ between the two jets as a function of the particle altitude.

$10 D$. For each force step, the simulation runs for $400 \mathrm{~s}$ with a time resolution of $5 \mathrm{~ms}$. The sphere is initially in the left jet.

Figure 11 (a) shows the probability density map of the sphere. The configuration of the jets is identical to the experimental configuration presented in figure2(a). The dashed lines indicated in the figure are the same as in figure 2 (a). The solid red line in figure11 (a) is the maximum of the probability map for each line. It is obtained with the same analysis used with the experimental data.

Figure 11(b) shows the probability of presence for different distances to the nozzle corresponding to the force steps $F / F_{b}=5.4,5.8,6.2,6.6,7.0,7.4,7.8$ and 8.2. The first 3 steps are below the merging of the two equilibrium positions and the PDF is not maximal at the center of the jets $x=0$. The 4 highest altitudes, the PDF are maximal at $x=0$.

Figure 11 (c) is the transition rate $\Gamma$ as a function of the vertical distance to the nozzles. The switching probability is computed by counting the number of sign changes for the trajectory $x(t)$. Below the first dashed line, the switching probability is smaller than $0.05 \mathrm{~s}^{-1}$ which means that the switching from one jet to the other may happen after $20 \mathrm{~s}$ typically. This is consistent with the fact that almost no spontaneous transitions are observed in this region for the experiment presented in figure 2 (a). Between the two dashed lines, $\Gamma$ is of the order of $1 \mathrm{~s}^{-1}$ which means that the spontaneous switching is occurring many times during an acquisitions of a few minutes typically. After the second dashed line, the potential is a single well centered at $x=0$. The transition rate $\Gamma$ saturates at $4.1 \mathrm{~s}^{-1}$. This value is consistent with the fact the transition frequency cannot exceed the horizontal frequency of the single well $f_{S W} \sim 5$ $\mathrm{Hz}$.

\section{Particle dynamics in the asymmetric configuration: transition probability}

The equations of motion are solved in the asymmetric case with a force $\rho \mathcal{P}_{L}$ for the left jet and $\rho \mathcal{P}_{R}$ for the right jet. The total input force $\rho \mathcal{P}_{L}+\rho \mathcal{P}_{R}$ is varied up and down between a low value $F_{b} / F=3.6$ and a high value $F_{b} / F=7$. The ball is initially above the right jet. Figure 12 presents the cumulative probability for the particle to have transited to left jet at the end of the cycle as a function of the jets asymmetry $\mathcal{P}_{L} / \mathcal{P}_{R}$.

The transition probability is computed over 80 realizations of particle simulations for each value $\mathcal{P}_{L} / \mathcal{P}_{R}$. $\mathcal{P}_{L} / \mathcal{P}_{R}=1$ corresponds to a probability of the order of 0.5 as expected for a symmetric configuration of the double trap. The transition probability is adjusted to a fit $\left(\tanh \left(\left(\mathcal{P}_{L} / \mathcal{P}_{R}-1\right) / \epsilon_{0}\right)+1\right) / 2$ in which $\epsilon_{0}=0.118$ is the only adjustable parameter. This fit means that the transition probability goes to 1 exponentially with the distance to $\mathcal{P}_{L} / \mathcal{P}_{R}=1$ with a characteristic distance $\mathcal{P}_{L} / \mathcal{P}_{R}=0.118$. For instance, the transition is observed with a probability larger than $99 \%$ for $\mathcal{P}_{L} / \mathcal{P}_{R}>1.27$. 


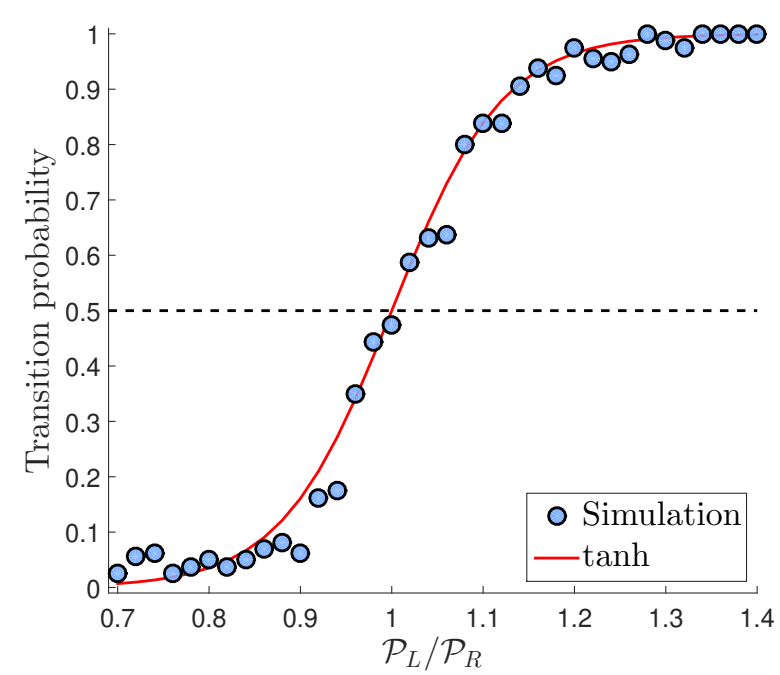

FIG. 12. Simulation results for the probability to transit from the right jet to the left jet as a function of the double jet asymmetry. For each data point, the sphere is initially in the right jet and the input force of the jet $\mathcal{P}_{L}+\mathcal{P}_{R}$ is varied up and down. The simulation is run 80 times for each ratio $\mathcal{P}_{L} / \mathcal{P}_{R}$ to compute an average number of transitions. For low $\mathcal{P}_{L} / \mathcal{P}_{R}$, the asymmetry corresponds to the situation in figure 9 for which the sphere in the right branch has little chance to transit to the left branch. For $\mathcal{P}_{L} / \mathcal{P}_{R}=1$, the configuration is symmetric and the probability to transit is close to $1 / 2$. A fit with a hyperbolic tangent $\left(\tanh \left(\left(\mathcal{P}_{L} / \mathcal{P}_{R}-1\right) / \epsilon_{0}\right)+1\right) / 2$ is performed with $\epsilon_{0}=0.118$.

\section{CONCLUSION}

The levitation of a sphere above two parallel and vertical turbulent jets realizes a transition from double-well levitation to single-well levitation. For an equal flow rate in the two nozzles, the transition is a symmetric pitchfork bifurcation controlled by the input flow rate. For different flow rates in the two nozzles, the bifurcation is asymmetric and a biased transition towards the jet of higher flow rate is observed.

Because the jets are turbulent, the velocity fluctuations are inducing a source of noise with observable effects on the bifurcation maps. In the symmetric configuration, the transition between the two stable equilibrium positions is possible and its transition rate depends on the distance to the bifurcation point. In the asymmetric configuration, the transition to the jet of higher flow rate is not systematic and fluctuations may induce transitions from the low energy well to the higher energy well.

We were not able to identify dynamical instabilities of the particle in levitation, mostly because the level of noise is high. If the existence of negative feedback processes cannot be ruled out, one should perform a more detail investigation of the effective damping of the particle to track the influence of such mechanisms.

A prediction of the force field with two jets is proposed with a simple summation of the force from each jet and using the measured force field for a single jet. This force field allows to predict the trapping map for different nozzle separations and also to carry out particle simulations including the effect of noise induced by the turbulent fluctuations.

In the context of particle contactless transport and manipulation using the levitation by turbulent jets, this work allows to quantify a first step with a double jet configuration. In particular, the numerical simulation can be used to (i) predict the altitude range for which the levitation above one of the jets is permanent and (ii) quantify which bias is necessary between the two adjacent jets for the transition to the jet of higher flow rate to occur.

\section{Appendix A: Force field: Empirical model 1 jet}

There is no general model to compute the drag and the lift exerted on a sphere for an arbitrary velocity profile. The object of this section is to establish an empirical formulation that relates a jet velocity profile and the resulting forces acting on a large sphere. 


\section{Measurement}

We consider a turbulent jet aligned with the $z$ axis with its origin in $x=0, y=0$ and $z=0$. The force acting on a sphere of diameter $D=40 \mathrm{~mm}$ in the turbulent jet is measured with the same strategy used for the two jets presented in section IVA. The force components $F_{x}$ and $F_{z}$ are presented in figure 13 (a) and (c) respectively. Figure 13 (b) and (d) are the empirical fitting profiles described below.

(a)

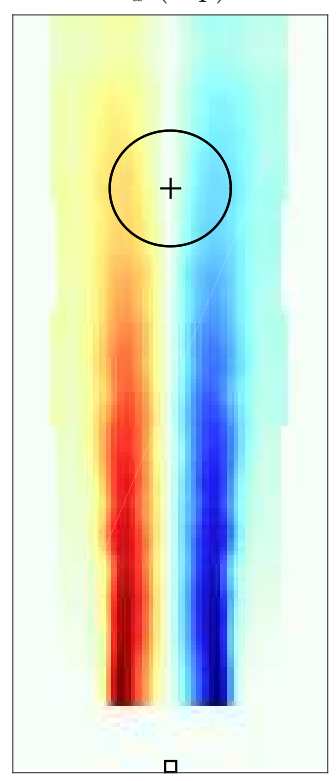

$F_{x}$ (model)

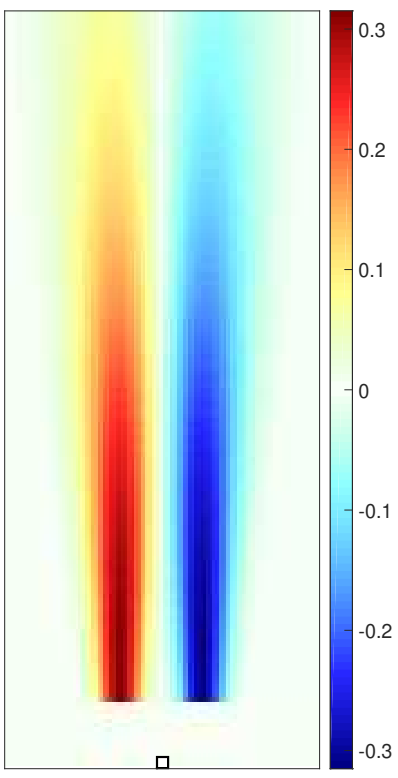

(b)

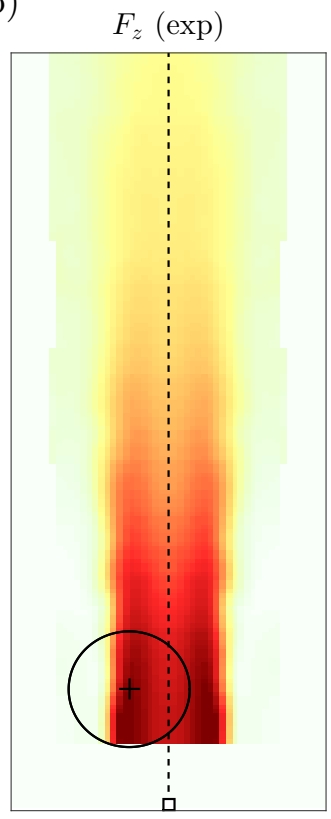

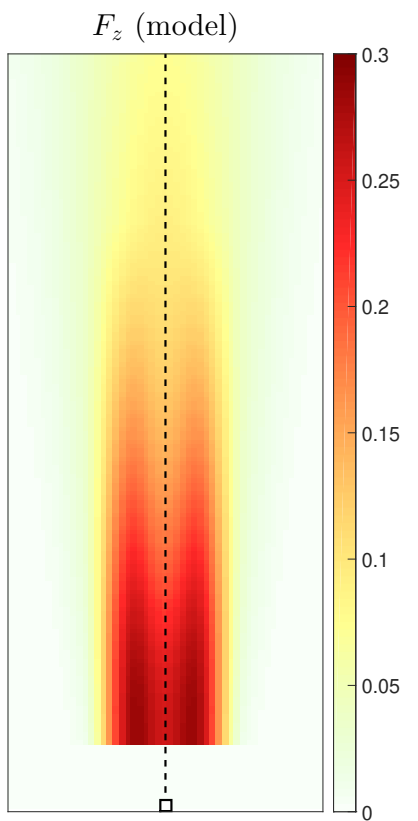

FIG. 13. (a) Horizontal force exerted on a sphere by a turbulent jet. The map on the left is the force measured with the sphere placed on a cantilever. The sphere of diameter $40 \mathrm{~mm}$ is represented at a stable equilibrium position. The map on the right is the model in eq. (A2). (b) Vertical force with measurements on the left and model given eq. (A1) on the right. The sphere is represented at a position where the vertical force is maximal. This position is not an equilibrium position.

\section{Modal decomposition}

In order to describe the forces acting on a sphere of diameter $D$ a modal decomposition is used ${ }^{36}$ in which the axial force (drag) and the horizontal force (lift) are given by:

$$
\begin{aligned}
& F_{z}(x, z)=\mathcal{Z}_{0}\left(\frac{z}{D}\right) \phi_{0}\left(\frac{x}{z-\delta_{z}}\right)+\mathcal{Z}_{1}\left(\frac{z}{D}\right) \phi_{1}\left(\frac{x}{D}\right) \\
& F_{x}(x, z)=\mathcal{X}_{0}\left(\frac{z}{D}\right)\left(\psi_{0}\left(\frac{x+\Delta x}{z-\delta_{x}}\right)-\psi_{0}\left(\frac{x-\Delta x}{z-\delta_{x}}\right)\right)
\end{aligned}
$$

The mode $\mathcal{Z}_{0} \phi_{0}$ for $F_{z}$ has the same symmetry as the velocity profile of the turbulent jet. It corresponds to a Gaussian profile $\phi_{0}$ with an ad hoc function for the amplitude $\mathcal{Z}_{0}$ on the centerline:

$$
\begin{aligned}
\mathcal{Z}_{0}\left(\frac{z}{D}\right) & =\frac{1}{\left(1+e_{\mathcal{Z}_{0}}\left(\frac{z}{D}\right)^{\eta_{1}}\right)^{\eta_{2}}} \\
\phi_{0}\left(\frac{x}{z-\delta_{z}}\right) & =\exp \left(-\sigma_{\mathcal{Z}_{0}}\left(\frac{x}{z-\delta_{z}}\right)^{2}\right)
\end{aligned}
$$


For $F_{x}$, two Gaussian modes shifted by $\pm \Delta x$ are used to obtain an anti-symmetric function of $x$ :

$$
\begin{aligned}
\mathcal{X}_{0}(z) & =\frac{A_{\mathcal{X}_{0}}}{1+e_{\mathcal{X}_{0}}\left(\frac{z}{D}\right)^{\eta_{3}}} \\
\psi_{0}\left(\frac{x \pm \Delta x}{z-\delta_{x}}\right) & =\exp \left(-\sigma_{\mathcal{X}_{0}}\left(\frac{x \pm \Delta x}{z-\delta_{x}}\right)^{2}\right)
\end{aligned}
$$

The mode $\mathcal{Z}_{1} \phi_{1}$ is a near-field correction to account for the force profile close to the nozzle and notably the fact that $F_{z}$ is not maximal on the centerline:

$$
\begin{aligned}
\mathcal{Z}_{1}(z) & =A_{\mathcal{Z}_{1}} \frac{z_{\phi_{1}}-z}{D} \\
\phi_{1}(x, z) & =\sin ^{2}\left(2 \pi \lambda_{\phi_{1}} \frac{x}{D}\right)
\end{aligned}
$$

The amplitudes $\mathcal{X}_{0}(z)$ and $\mathcal{Z}_{0}(z)$ are adjusted by extracting the maximum for $F_{x}$ and $F_{z}$ for at a given altitude $z$. The results of the fit are presented in figure 14 (a) and (b).

(a)

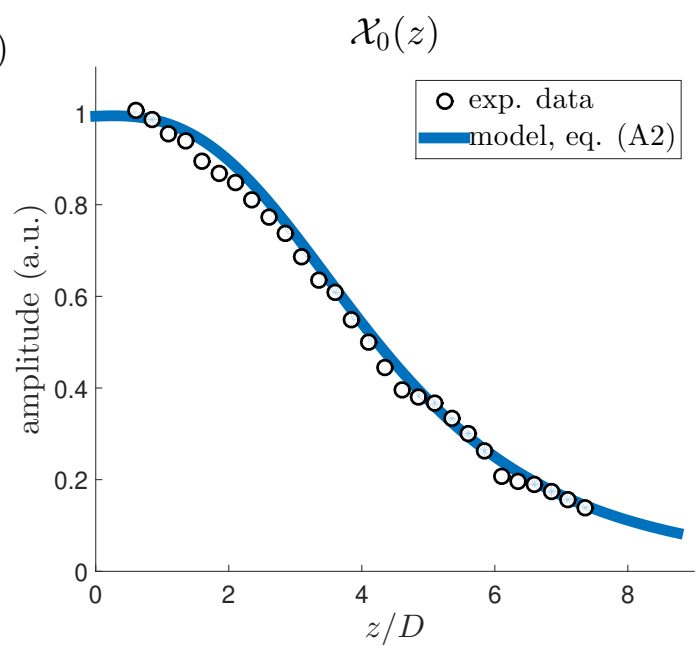

(b)

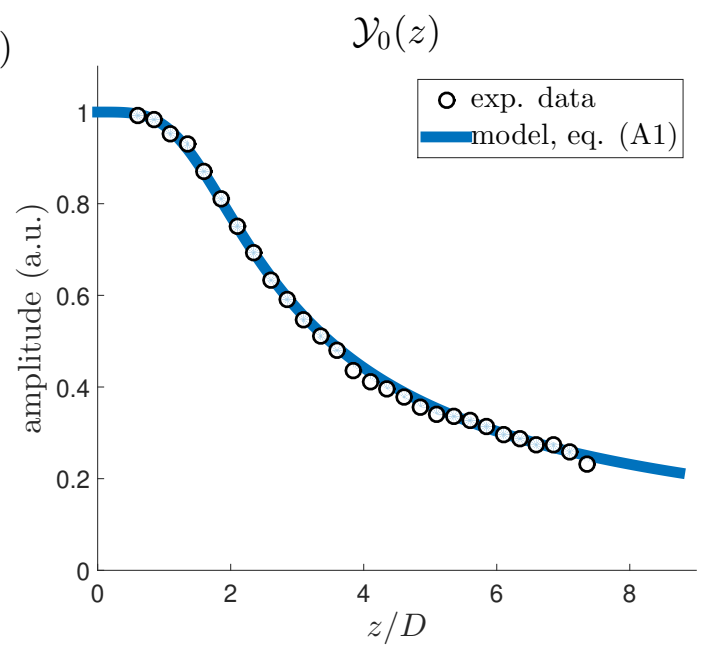

FIG. 14. (a) Amplitude of the mode for the horizontal component of the force as a function of the vertical distance to the nozzle. The fitting function is given by eq. A5. (b) Amplitude of the mode for the vertical component of the force. The fitting function is given by eq. (A33).

\begin{tabular}{|c|c|c|c|c|c|c|c|c|c|c|c|}
\hline mode & \multicolumn{3}{|c|}{$\mathcal{Z}_{0} \phi_{0}$} & & $\mathcal{Z}_{1} \phi_{1}$ & & \multicolumn{5}{|c|}{$\mathcal{X}_{0} \phi_{0}$} \\
\hline arameter & $e_{\mathcal{Z}_{0}}$ & $\eta_{2}$ & $\sigma_{\mathcal{Z}_{0}} \delta_{z}(\mathrm{~mm})$ & $A_{\mathcal{Z}_{1}}$ & $z_{\phi_{1}}(\mathrm{~mm})$ & $\lambda_{\phi_{1}}$ & $A_{\mathcal{X}_{0}}$ & $e_{\mathcal{X}_{0}}$ & $\eta_{3}$ & $\sigma_{\mathcal{X}_{0}}$ & ${ }_{x}(\mathrm{~mm}) \Delta_{x}$ \\
\hline value & 0.1293 .84 & 0.246 & 179 & 0.130 & 201 & 0.751 & 1.243 & 0.0164 & 2.78 & 267 & 90.6 \\
\hline
\end{tabular}

A global optimization of the free parameters is performed by minimizing the absolute distance between the measured force maps in figure 13 (a) and (c) and the model force maps in figure (b) and (d) respectively. The values of the free parameters are presented in table 1

TABLE I. Fitting parameters of the modal decomposition for the forces exerted on a sphere by a turbulent jet.

Since a turbulent round jet has a cylindrical symmetry, equations (A1) and A2 with the values in table I can be used to describe a $3 \mathrm{D}$ force field $\left\{F_{x}(x, y, z), F_{y}(x, y, z), F_{z}(x, y, z)\right\}$ for a spherical particle in a turbulent jet. Moreover and because the jet has a self-similar structure, this force field is valid for spheres with different diameters using the scaled parameters $x / D, y / D$ and $z / D$.

\section{Appendix B: Calibration for the particle simulation}

The parameters corresponding to the linear damping and the noise term are calibrated for a single jet configuration. 


\section{Nature of the particle fluctuations}

A particle's trajectory is presented in figure 15 (a) and (b) with the horizontal $x(t)$ and the vertical $z(t)$ positions of a trapped particle in a double jet configuration. The flow rate is independent of time and corresponds to the PDF image $F_{j} / F=2.38$ in figure 4. The particle is in the regime (ii) with spontaneous jumps between 2 positions.

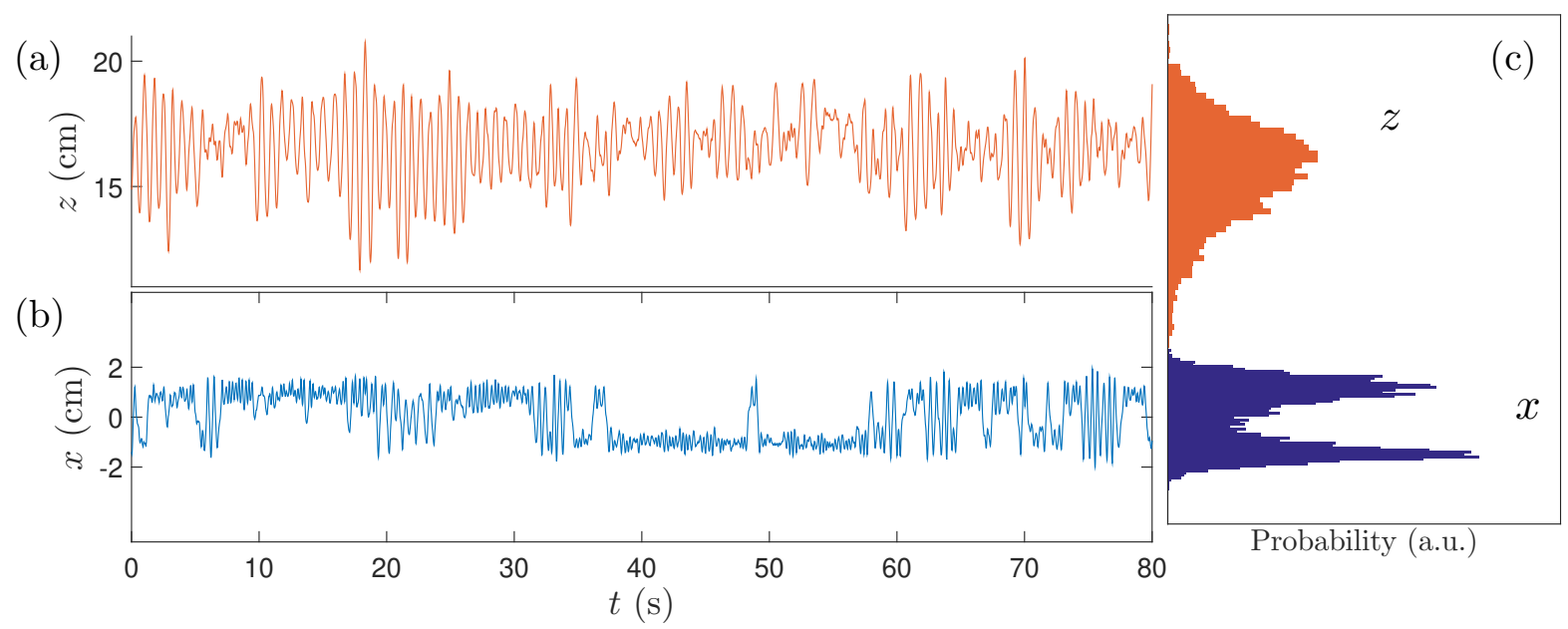

FIG. 15. Trajectories for the horizontal $x(t)$ and the vertical $z(t)$ motion of a sphere in levitation with two parallel turbulent jets. The flow rate is symmetric and such as the particle is in the regime (ii) (see figure 2) with spontaneous jumps between the two jets. $F_{j} / F=2.38$, see figure 4 The probability of presence are represented on the right.

The vertical position $z(t)$ corresponds to the fluctuation signal of a damped harmonic oscillator driven by a noise. The particle probability is presented in figure 15(c) and the fluctuations appear Gaussian.

For the horizontal position $x(t)$, the particle probability of presence is bi-modal because the particle jumps between the left and the right jet. Within the step, the signal seems to be noise-induced, even if it is less clear than for $z(t)$.

\section{Damping}

An acquisition of a particle trajectory is performed in a single turbulent jet with fixed flow rate corresponding to an equilibrium distance to the nozzle $z / D=3.88$. The ball is displaced vertically from its equilibrium position and the trajectory is recorded during $16 \mathrm{~s}$ at a frame rate of $40 \mathrm{fps}$. The trajectory of the horizontal and vertical component is presented in figure 16 (a).

The trajectory of a particle in single turbulent jet is computed for the analytical force field established in appendix A with a linear vertical damping of $\gamma / m=0.4 \mathrm{~s}^{-1}$ and without noise. The trajectory obtained is presented in figure 16 (b).

The natural frequencies for the horizontal and vertical oscillations are measured to $f_{x, \exp }=5.92 \mathrm{~Hz}$ and $f_{z, \exp }=1.43$ $\mathrm{Hz}$ in the experiment and $f_{x, s i m u}=6.31 \mathrm{~Hz}$ and $f_{z, \text { simu }}=1.19 \mathrm{~Hz}$ in the simulation.

\section{Noise}

A simulation of a particle trajectory is carried out by sweeping the magnitude of the jet from $\sqrt{F / F_{b}}=2.3$ to $\sqrt{F / F_{b}}=5.8$ with an added white noise $\eta_{i}(t)$.

The RMS displacement for the horizontal and vertical motion are represented as a function of the normalized distance $Z / D$ in figure 17. The random noise $\eta_{i}(t)$ is set to an amplitude of $5.8 \times 10^{-2} \mathrm{~m} \cdot \mathrm{s}^{-2} / \sqrt{\mathrm{Hz}}$ so that the results presented in the figure are consistent with the RMS displacements reported by Davoust and Jacquin ${ }^{6}$.

${ }^{1}$ E. Brandt, Science 243, 349 (1989).

${ }^{2}$ O. Reynolds, A. W. Brightmore, and W. H. Moorby, Papers on Mechanical and Physical Subjects: 1869-1882, Vol. 1 (The University Press, 1900).

${ }^{3}$ A. Frank, Russian Journal of Numerical Analysis and Mathematical Modelling 15, 145 (2000).

${ }^{4}$ J. Feng and D. Joseph, Journal of Fluid Mechanics 315, 367 (1996). 
(a)

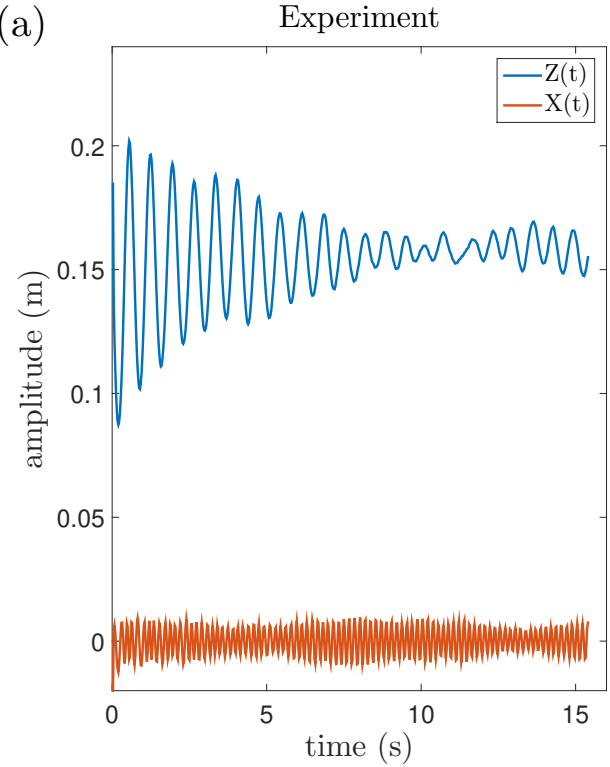

(b)

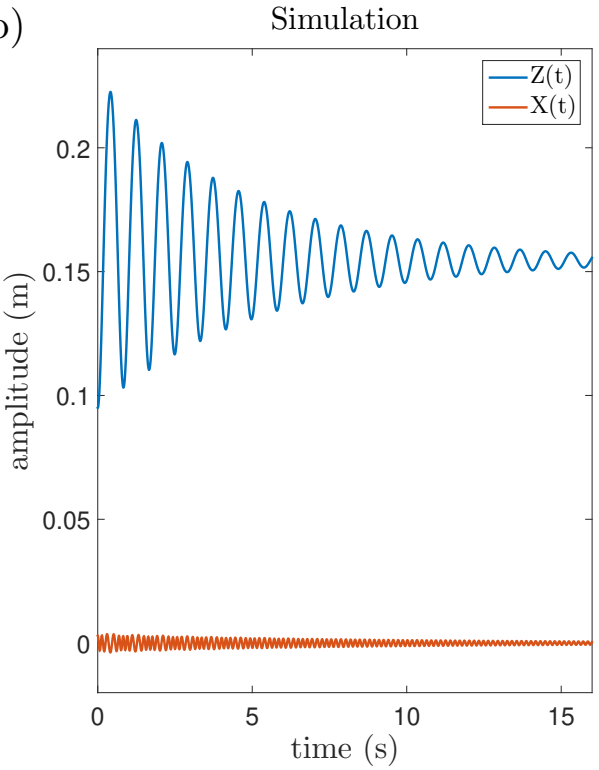

FIG. 16. (a) Trajectory of a sphere in levitation in a turbulent jet. (b) Simulated trajectory of a sphere using the set of equation (9), (10) and (11) with the value for the vertical damping $\gamma / m=0.4 \mathrm{~s}^{-1}$ and without noise $\eta_{i}(t)=0$.

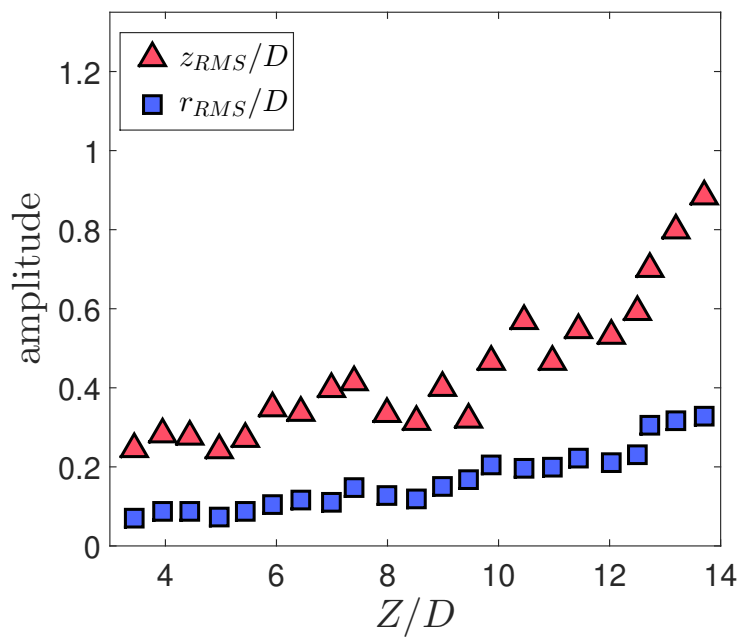

FIG. 17. Amplitude of the vertical and radial root mean square displacement of a sphere in levitation in a turbulent jet as a function of the vertical distance to the nozzle.

${ }^{5}$ K. T. McDonald, American Journal of Physics 68, 388 (2000).

${ }^{6} \mathrm{~S}$. Davoust and L. Jacquin, in TSFP DIGITAL LIBRARY ONLINE (Begel House Inc., 2009).

${ }^{7}$ A. Becker, R. Sandheinrich, and T. Bretl, in 2009 IEEE/RSJ International Conference on Intelligent Robots and Systems (IEEE, 2009) pp. 781-786.

${ }^{8}$ I. Reba, Scientific American 214, 84 (1966).

${ }^{9}$ T. López-Arias, L. Gratton, S. Bon, and S. Oss, The Physics Teacher 47, 508 (2009).

${ }^{10}$ T. Barois, P. Huck, M. Bourgoin, and R. Volk, Physical Review E 96, 033105 (2017).

${ }^{11}$ T. Barois, P. D. Huck, C. Paleo, M. Bourgoin, and R. Volk, Physics of Fluids 31, 125116 (2019).

${ }^{12}$ I. Wygnanski and H. Fiedler, Journal of Fluid Mechanics 38, 577 (1969).

${ }^{13}$ H. J. Hussein, S. P. Capp, and W. K. George, Journal of Fluid Mechanics 258, 31 (1994).

${ }^{14}$ G. Xu and R. Antonia, Experiments in fluids 33, 677 (2002).

${ }^{15} \mathrm{~J}$. Mi and G. Nathan, Flow, turbulence and combustion 84, 583 (2010).

${ }^{16}$ M. Announ, J. Badie, B. Granier, and P. Snabre, High Temperature Material Processes: An International Quarterly of High-Technology Plasma Processes 5 (2001).

${ }^{17}$ G. Mathiak, I. Egry, L. Hennet, D. Thiaudiere, I. Pozdnyakova, and D. Price, International journal of thermophysics 26, 1151 (2005). 
${ }^{18}$ A. Pack, K. Kremer, N. Albrecht, K. Simon, and A. Kronz, Geochemical transactions 11, 4 (2010).

${ }^{19}$ J. H. Snoeijer and K. v. der Weele, American journal of physics 82, 1029 (2014).

${ }^{20}$ R. Ojha, P.-A. Lemieux, P. Dixon, A. Liu, and D. Durian, Nature 427, 521 (2004).

${ }^{21}$ E. Fällman and O. Axner, Applied Optics 36, 2107 (1997).

${ }^{22}$ L. I. McCann, M. Dykman, and B. Golding, Nature 402, 785 (1999).

${ }^{23}$ A. Stilgoe, N. Heckenberg, T. Nieminen, and H. Rubinsztein-Dunlop, Physical review letters 107, 248101 (2011).

${ }^{24}$ L. Rondin, J. Gieseler, F. Ricci, R. Quidant, C. Dellago, and L. Novotny, Nature nanotechnology 12, 1130 (2017).

${ }^{25}$ J. Gladrow, M. Ribezzi-Crivellari, F. Ritort, and U. F. Keyser, Nature communications 10, 1 (2019).

${ }^{26}$ S. H. Strogatz, Nonlinear dynamics and chaos: with applications to physics, biology, chemistry, and engineering (CRC Press, 2018).

${ }^{27}$ T. López-Arias, L. Gratton, G. Zendri, and S. Oss, Physics Education 46, 146 (2011).

${ }^{28}$ H. Reichardt, The Aeronautical Journal 47, 167 (1943).

${ }^{29}$ L. G. Alexander, T. Baron, and E. W. Comings, "Transport of momentum, mass, and heat in turbulent jets," Tech. Rep. (University of Illinois at Urbana Champaign, College of Engineering ..., 1953).

${ }^{30}$ R. Knystautas, The Aeronautical Quarterly 15, 1 (1964).

${ }^{31}$ B. Pani and P. Parameswaran, Journal of Hydraulic Research 32, 53 (1994).

${ }^{32}$ J. E. Hodgson, A. K. Moawad, and N. Rajaratnam, Journal of Hydraulic Research 37, 249 (1999).

${ }^{33}$ B. S. Pani, Water Science and Engineering 2, 19 (2009).

${ }^{34}$ P. C. Yannopoulos and G. C. Noutsopoulos, Journal of Hydraulic Research 44, 233 (2006).

${ }^{35}$ A. C. Lai and J. H. Lee, Journal of fluid mechanics 708, 539 (2012).

${ }^{36}$ R. Neve, R. Nelson, and P. Kotsiopoulos, Journal of Fluid Mechanics 107, 521 (1981). 